\title{
Reinforcement Belief Revision
}

\author{
Yi Jin \\ School of Computing Science \\ Simon Fraser University \\ yijecs.sfu.ca \\ Michael Thielscher \\ Department of Computer Science \\ Dresden University of Technology \\ mit@inf.tu-dresden.de
}

\begin{abstract}
The capability of revising its beliefs upon new information in a rational and efficient way is crucial for an intelligent agent. The classical work in belief revision focuses on idealized models and is not concerned with computational aspects. In particular, many researchers are interested in the logical properties (e.g., the AGM postulates) that a rational revision operator should possess. For the implementation of belief revision, however, one has to consider that any realistic agent is a finite being and that calculations take time. In this paper, we introduce a new operation for revising beliefs which we call reinforcement belief revision. The computational model for this operation allows us to assess it in terms of time and space consumption. Moreover, the operation is proved equivalent to a (semantical) model based on the concept of possible worlds, which facilitates showing that reinforcement belief revision satisfies all desirable rationality postulates.
\end{abstract}

\section{Introduction}

Belief revision is the process of adapting the beliefs of an agent to accommodate new, more precise, or more reliable information that is possibly inconsistent with the existing beliefs. The formal study of belief revision took as starting point the work of Alchourrón, Gärdenfors, and Makinson (AGM) during the first half of the 1980s [Alchourrón et al., 1985, Alchourrón and Makinson, 1985]. The AGM framework is an idealized mathematical model of belief revision: It assumes that the beliefs of an agent are represented by a so-called belief set, a logically closed set of sentences in some underlying language $\mathcal{L}$. The new evidence is also represented by a sentence in $\mathcal{L}$, and a revision operator is then modeled as a function mapping the current belief set and the new evidence to a revised belief set.

To provide general design criteria for belief revision operators, the AGM trio developed a set of so-called rationality postulates [Alchourrón et al., 1985]. The guiding principle of the AGM postulates is that of economy of information or minimal change, which means to not give up currently held beliefs and not generate new beliefs unless necessary. As pointed out by several researchers Gärdenfors and Makinson, 1988 Spohn, 1988], the AGM postulates do not uniquely determine a revision operator, and 
revision operators should, in general, exploit extra-logical information concerning the preference (or plausibility) over different beliefs in order to determine the revision strategy. The Triviality Theorem of [Gärdenfors, 1988] shows that, when accepting the AGM postulates, it is improper to include extra-logical preference information into the belief sets. As a consequence, we need to distinguish a belief set from a belief state (also called epistemic state). The latter contains, in addition to its belief set, the extralogical preference information (also referred to as conditional beliefs [Boutilier, 1993]) which determines the revision strategy. Like in [Darwiche and Pearl, 1997|, we will therefore consider revision operators to be functions on belief states in this paper.

For the incremental adaptation of beliefs, the AGM postulates proved to be too weak [Darwiche and Pearl, 1997] due to the excessive freedom they permit on the change of the conditional beliefs. This has led to the development of additional postulates for iterated belief revision by Darwiche and Pearl (DP), among others. Still, however, the AGM and DP postulates together are too permissive in that they support belief revision operators which assume arbitrary dependencies among the pieces of information which an agent acquires along its way. These dependencies have a drastic effect when the agent makes an observation which contradicts its currently held beliefs: The agent is forced to cancel everything it has learnt up to this point |Nayak et al., 1996, Nayak et al., 2003|. To handle dependencies properly, we have recently proposed the postulate of Independence as a complement to the AGM+DP postulates [Jin and Thielscher, 2007].

In classical belief revision, the agents are considered to have unlimited memory, time, and deductive ability. For implementing belief revision, however, one has to consider that any realistic agent is a finite being and that calculations take time. Therefore, the beliefs of a realistic agent should always be finitely representable; and a revision operator should not only behave rationally but also consume a reasonable amount of time and space. Arguably, adapting belief revision to realisitic settings is far from trivial, as we need an approach which takes these characteristics of finiteness as well as memory and time limitations into account [Wassermann, 1999].

In this paper, we introduce a concrete belief revision operator, called reinforcement belief revision. Specifically, we present a computational model for reinforcement revision which operates on finite sets of integer-weighted sentences. This operation is proved equivalent to a possible world-based (semantical) model, which enables us to show that reinforcement belief revision satisfies the desirable rationality postulates. In addition, we will analyze the computational complexity of reinforcement belief revision. Finally, we will place our result in the context of possibility theory, which is a field of research that is intimately related to belief revision Dubois and Prade, 1992. Dubois et al., 1994|: In |Benferhat et al., 2002], for instance, it is shown that some well-known revision operators can be directly mapped to well-known conditioning operations in possibility theory. In this paper, we will likewise show how reinforcement belief revision can be re-cast in the possibilistic setting. The result is a novel approach to revising possibilistic knowledge states.

The rest of the paper is organized as follows. In the next section, we recall the classical AGM postulates, followed by postulates for iterated revision. In Section 3 we introduce reinforcement belief revision by giving both a semantical and a computational definition. Reinforcement belief revision is assessed in terms of its logical properties and computational complexity. In Section 4 we re-model reinforcement belief revision in the setting of possibility theory. We conclude in Section 5 with a detailed comparison to related work. Proofs of the main results can be found in the appendix. 


\section{Preliminaries}

In this paper, we will deal with a finitary propositional language $\mathcal{L}$ generated from a finite set $\mathcal{P}$ of atomic propositions. It is assumed that $\mathcal{L}$ is equipped with the classical consequence relation $\vdash$. Given a set $S$ of sentences, $C n(S)$ consists of all logical consequences of $S$, that is $C n(S)=\{\alpha \in \mathcal{L} \mid S \vdash \alpha\}$. Two sentences $\alpha$ and $\beta$ are logically equivalent, written $\alpha \equiv \beta$, iff $\alpha \in C n(\{\beta\})$ and $\beta \in C n(\{\alpha\})$. A propositional interpretation (also referred to as a possible world) is a mapping from $\mathcal{P}$ to $\{\top, \perp\}$, and the set of all interpretations is denoted by $\Theta_{\mathcal{L}}$. For the sake of simplicity, we may represent an interpretation by the set of atoms to which it assigns $\top$. An interpretation $W$ is called a model of $S$ (denoted by $W \models S$ ) if it truth functionally maps all sentences in $S$ to $\top$. The set of all models of $S$ is denoted by $\operatorname{Mods}(S)$. Conversely, given a set $\mathcal{W}$ of possible worlds, $\operatorname{Th}(\mathcal{W})$ denotes the set of all sentences which are true in each element of $\mathcal{W}$. For easier readability, in the rest of the paper we will often identity a singleton set with its element. For instance, $\operatorname{Mods}(\{\alpha\})$ may also be written as $\operatorname{Mods}(\alpha)$.

A belief set $K$ is a logically closed set of sentences, that is, $K=C n(K)$. The expansion of a belief set $K$ by a sentence $\alpha$ is defined as: $K+\alpha \stackrel{\text { def }}{=} C n(K \cup\{\alpha\})$. For the sake of generality, we deliberately consider a belief state $\mathcal{K}$ as an abstract object from which we can derive a belief set, denoted by $\operatorname{Bel}(\mathcal{K})$, and also some extra-logical preference information. In concrete constructions of revision operators, the extra-logic preference information could take the form of a relation over the set of all sentences, or a relation over all possible worlds, etc. Moreover, we say that two belief states $\mathcal{K}_{1}, \mathcal{K}_{2}$ are logically equivalent (written as $\mathcal{K}_{1} \equiv \mathcal{K}_{2}$ ) iff $\operatorname{Bel}\left(\mathcal{K}_{1}\right)=\operatorname{Bel}\left(\mathcal{K}_{2}\right)$.

A total pre-order $\leq$ (possibly indexed) is a reflexive, transitive, binary relation, such that for any $\alpha, \beta$ : either $\alpha \leq \beta$ or $\beta \leq \alpha$. The strict part of $\leq$ is denoted by $<$, that is, $\alpha<\beta$ iff $\alpha \leq \beta$ and $\beta \not \leq \alpha$. As usual, $\alpha=\beta$ abbreviates $\alpha \leq \beta$ and $\beta \leq \alpha$. Given a total pre-order $\leq$ on $S$, we denote by $\min (S, \leq)$ the set of all minimal elements of $S$ wrt. $\leq$. Natural numbers and positive integers (i.e., natural number greater than 0 ) are denoted by $\mathbb{N}$ and $\mathbb{N}^{+}$, respectively. The cardinality of a set $S$ is denoted by $\|S\|$, and the size of $S$ (the number of symbols occurring in $S$ ) is denoted by $|S|$,

\subsection{The AGM Postulates}

The AGM postulates Alchourrón et al., 1985 provide a mathematical foundation for belief revision by defining criteria for rational revision operators. The original postulates have been reformulated in [Darwiche and Pearl, 1997| as follows, where $\mathcal{K} * \alpha$ denotes the belief state resulting from the revision of belief state $\mathcal{K}$ by sentence $\alpha$ :

$$
\begin{array}{ll}
(\mathcal{K} * 1) & \operatorname{Bel}(\mathcal{K} * \alpha)=\operatorname{Cn}(\operatorname{Bel}(\mathcal{K} * \alpha)) \\
(\mathcal{K} * 2) & \alpha \in \operatorname{Bel}(\mathcal{K} * \alpha) \\
(\mathcal{K} * 3) & \operatorname{Bel}(\mathcal{K} * \alpha) \subseteq \operatorname{Bel}(\mathcal{K})+\alpha \\
(\mathcal{K} * 4) & \text { If } \neg \alpha \notin \operatorname{Bel}(\mathcal{K}) \text { then } \operatorname{Bel}(\mathcal{K})+\alpha \subseteq \operatorname{Bel}(\mathcal{K} * \alpha) \\
(\mathcal{K} * 5) & \operatorname{Bel}(\mathcal{K} * \alpha) \text { is inconsistent only if } \vdash \neg \alpha \\
(\mathcal{K} * 6) & \text { If } \alpha \equiv \beta \text { then } \mathcal{K} * \alpha \equiv \mathcal{K} * \beta
\end{array}
$$




$$
\begin{array}{ll}
(\mathcal{K} * 7) & \operatorname{Bel}(\mathcal{K} *(\alpha \wedge \beta)) \subseteq \operatorname{Bel}(\mathcal{K} * \alpha)+\beta \\
(\mathcal{K} * 8) & \text { If } \neg \beta \notin \operatorname{Bel}(\mathcal{K} * \alpha) \text { then } \operatorname{Bel}(\mathcal{K} * \alpha)+\beta \subseteq \operatorname{Bel}(\mathcal{K} *(\alpha \wedge \beta))
\end{array}
$$

Readers are referred to Gärdenfors and Makinson, 1988 for a detailed account of the motivation and intuition behind these postulates.

Various approaches to constructing AGM revision operators have been proposed in the literature. Here, we will only sketch the basic ideas of two of them which are crucial to the rest of the paper.

\subsubsection{Epistemic Entrenchment}

Gärdenfors and Makinson, 1988] have suggested to represent the extra-logical preference information of a belief state by a total pre-order on the underlying language $\mathcal{L}$. Formally, an epistemic entrenchment $\leq_{K}$ on a belief set $K$ is a binary relation over $\mathcal{L}$ satisfying the following conditions:

$$
\begin{aligned}
& \text { (EE1) If } \alpha \leq_{K} \beta \text { and } \beta \leq_{K} \gamma \text {, then } \alpha \leq_{K} \gamma \\
& \text { (EE2) } \quad \text { If } \alpha \vdash \beta \text {, then } \alpha \leq_{K} \beta \\
& \text { (EE3) } \quad \text { For any } \alpha \text { and } \beta, \alpha \leq_{K} \alpha \wedge \beta \text { or } \beta \leq_{K} \alpha \wedge \beta \\
& \text { (EE4) } \quad \text { When } K \text { is consistent, } \alpha \notin K \text { iff } \alpha \leq_{K} \beta \text { for all } \beta \\
& \text { (EE5) If } \beta \leq_{K} \alpha \text { for all } \beta \text {, then } \vdash \alpha
\end{aligned}
$$

Intuitively, $\alpha \leq_{K} \beta$ means that $\beta$ is at least as plausible as $\alpha$.

It has been shown by Gärdenfors and Makinson, 1988 that the AGM postulates characterize exactly the class of epistemic entrenchment-based revision operators: A revision operator $*$ satisfies $(\mathcal{K} * 1)-(\mathcal{K} * 8)$ iff for any belief state $\mathcal{K}$ there exists an epistemic entrenchment $\leq_{\operatorname{Bel}(\mathcal{K})}$ on $\operatorname{Bel}(\mathcal{K})$, such that for any sentence $\alpha$ :

$$
\operatorname{Bel}(\mathcal{K} * \alpha)= \begin{cases}\mathcal{L} & \text { if } \vdash \neg \alpha \\ \left\{\beta \in \mathcal{L} \mid \neg \alpha<_{B e l(\mathcal{K})} \neg \alpha \vee \beta\right\} & \text { otherwise }\end{cases}
$$

\subsubsection{Faithful Ranking}

From quite a different perspective, |Katsuno and Mendelzon, 1991] have proposed a constructive model for belief revision based on possible worlds. Formally, a faithful ranking $\preceq_{K}$ on a belief set $K$ is a total pre-order on the set of all possible worlds $\Theta_{\mathcal{L}}$, such that for any possible worlds $W_{1}, W_{2}$ :

1. If $W_{1}, W_{2} \models K$, then $W_{1}={ }_{K} W_{2}$

2. If $W_{1} \models K$ and $W_{2} \not \models K$, then $W_{1} \prec_{K} W_{2}$

The intuitive meaning of $W_{1} \preceq_{K} W_{2}$ is that $W_{1}$ is at least as plausible as $W_{2}$.

Similar to epistemic entrenchment-based revision, |Katsuno and Mendelzon, 1991 have shown that the AGM postulates characterize exactly the class of faithful rankingbased revision operators: A revision operator $*$ satisfies $(\mathcal{K} * 1)-(\mathcal{K} * 8)$ iff for any belief state $\mathcal{K}$ there exists a faithful ranking $\preceq_{\operatorname{Bel}(\mathcal{K})}$ on $\operatorname{Bel}(\mathcal{K})$ such that for any sentence $\alpha$ :

$$
\operatorname{Bel}(\mathcal{K} * \alpha)= \begin{cases}\mathcal{L} & \text { if } \vdash \neg \alpha \\ \operatorname{Th}\left(\min \left(\operatorname{Mods}(\alpha), \preceq_{\operatorname{Bel}(\mathcal{K})}\right)\right) & \text { otherwise }\end{cases}
$$




\subsection{Postulates for Iterated Revision}

\subsubsection{The DP Postulates}

It is well-known that the AGM postulates sanction improper response to sequences of new information due to the excessive freedom they allow on the change of the conditional beliefs. Therefore, [Darwiche and Pearl, 1997] have proposed four additional postulates for iterated belief revision:

$$
\begin{aligned}
& \text { (DP1) } \quad \text { If } \beta \vdash \alpha \text {, then } \operatorname{Bel}((\mathcal{K} * \alpha) * \beta)=\operatorname{Bel}(\mathcal{K} * \beta) \\
& \text { (DP2) } \quad \text { If } \beta \vdash \neg \alpha \text {, then } \operatorname{Bel}((\mathcal{K} * \alpha) * \beta)=\operatorname{Bel}(\mathcal{K} * \beta) \\
& \text { (DP3) } \quad \text { If } \alpha \in \operatorname{Bel}(\mathcal{K} * \beta) \text {, then } \alpha \in \operatorname{Bel}((\mathcal{K} * \alpha) * \beta) \\
& \text { (DP4) } \quad \text { If } \neg \alpha \notin \operatorname{Bel}(\mathcal{K} * \beta), \text { then } \neg \alpha \notin \operatorname{Bel}((\mathcal{K} * \alpha) * \beta)
\end{aligned}
$$

A detailed account of the motivation and interpretation of these postulates can be found in |Darwiche and Pearl, 1997|.

To provide formal justifications, [Darwiche and Pearl, 1997] have also given a nice representation result for Postulates (DP1)-(DP4): Let $*$ be a revision operator satisfying Postulates $(\mathcal{K} * 1)-(\mathcal{K} * 8)$, then $*$ satisfies Postulates (DP1)-(DP4) iff the corresponding faithful rankings satisfy the following conditions 1

$$
\begin{array}{r}
\text { (DPR1) If } W_{1}, W_{2} \models \alpha \text {, then } W_{1} \preceq_{\mathcal{K}} W_{2} \text { iff } W_{1} \preceq_{\mathcal{K} * \alpha} W_{2} \\
\text { (DPR2) If } W_{1}, W_{2} \not \models \alpha, \text { then } W_{1} \preceq \mathcal{K} W_{2} \text { iff } W_{1} \preceq \mathcal{K}_{* \alpha} W_{2} \\
\text { (DPR3) If } W_{1} \models \alpha \text { and } W_{2} \not \models \alpha, \text { then } W_{1} \prec_{\mathcal{K}} W_{2} \text { implies } \\
W_{1} \prec_{\mathcal{K} * \alpha} W_{2} \\
\text { (DPR4) If } W_{1} \models \alpha \text { and } W_{2} \not \models \alpha, \text { then } W_{1} \preceq_{\mathcal{K}} W_{2} \text { implies } \\
W_{1} \preceq \mathcal{K}_{* \alpha} W_{2}
\end{array}
$$

This result gives an elegant characterization of the seemingly natural constraints that the DP postulates impose on the change of the conditional beliefs: When $\mathcal{K}$ is revised by $\alpha$, Conditions (DPR1) and (DPR2) require to retain the relative plausible ordering of any two $\alpha$-worlds ( $\neg \alpha$-worlds, respectively); Conditions (DPR3) and (DPR4) require that if an $\alpha$-world $W_{1}$ is (strictly) more plausible than a $\neg \alpha$-world $W_{2}$, then $W_{1}$ continues to be (strictly) more plausible than $W_{2}$.

\subsubsection{Two Radical Revision Operators}

The AGM and DP postulates together still do not uniquely determine a revision operator. In the following, we present two interesting operators known from the literature which satisfy all AGM/DP postulates.

Boutilier, 1993 has proposed a specific revision operator (known as natural revision) which satisfies the following:

$$
\text { If } \neg \beta \in \operatorname{Bel}(\mathcal{K} * \alpha) \text {, then } \operatorname{Bel}((\mathcal{K} * \alpha) * \beta)=\operatorname{Bel}(\mathcal{K} * \beta)
$$

\footnotetext{
${ }^{1}$ For the sake of simplicity, $\preceq_{B e l(\mathcal{K})}$ and $\preceq_{B e l(\mathcal{K} * \alpha)}$ are abbreviated by $\preceq_{\mathcal{K}}$ and $\preceq \mathcal{K} * \alpha$, respectively
} 
This postulate alone is in fact a strengthening of the set of DP postulates, in the sense that (CB) implies all of them (in the presence of the AGM postulates) but not vice versa.

A semantical characterization for (CB) has been given in |Darwiche and Pearl, 1997|:

(CBR) If $W_{1}, W_{2} \forall \forall \operatorname{Bel}(\mathcal{K} * \alpha)$, then $W_{1} \preceq_{\mathcal{K}} W_{2}$ iff $W_{1} \preceq \mathcal{K}_{* \alpha} W_{2}$

Since Condition (CB) forces the agent to cancel the previous observation upon any new observation which contradicts its currently held beliefs, it has been criticized as too radical to serve as a general rationality postulate INayak et al., 2003. Jin and Thielscher, 2007|.

So-called lexicographic revision (with "naked evidence") satisfies the postulate of Recalcitrance |Nayak, 1994, Nayak et al., 2003|:

$(\operatorname{Rec})$

$$
\text { If } \beta \nvdash \neg \alpha \text {, then } \alpha \in \operatorname{Bel}((\mathcal{K} * \alpha) * \beta)
$$

Note that, in the presence of Postulates $(\mathcal{K} * 1)-(\mathcal{K} * 8)$, we can derive (DP3) and (DP4) from (Rec), but neither (DP1) nor (DP2).

The semantical characterization of (Rec) is as follows [Nayak et al., 2003]:

(RecR) If $W_{1} \models \alpha$ and $W_{2} \models \neg \alpha$, then $W_{1} \prec \mathcal{K}_{* \alpha \alpha} W_{2}$

As shown in [Konieczny and Pérez, 2000|, Postulate (Rec) is only suitable when the agent has full confidence in the new information; hence it, too, cannot serve as a general rationality postulate (cf. [Jin and Thielscher, 2007] for a counterexample).

\subsubsection{Postulate of Independence}

While both $(\mathrm{CB})$ and $(\mathrm{Rec})$ are too radical, the DP postulates alone are too weak because they do not address the problem of implicit dependence [Jin and Thielscher, 2007]. The additional postulate of Independence has been proposed to overcome this weakness:

(Ind) If $\neg \alpha \notin \operatorname{Bel}(\mathcal{K} * \neg \beta)$ then $\alpha \in \operatorname{Bel}((\mathcal{K} * \alpha) * \neg \beta)$

Note that (Ind) is a weakening of (Rec), while it is still strong enough to imply (DP3) and (DP4). Readers are referred to [Jin and Thielscher, 2007] for a detailed discussion on the problem of implicit dependence, and the motivation and interpretation of Postulate (Ind).

The semantical characterization for Postulate (Ind) is as follows:

(IndR) $\quad$ If $W_{1} \models \alpha$ and $W_{2} \models \neg \alpha$, then $W_{1} \preceq \mathcal{K} W_{2}$ implies

$$
W_{1} \prec{ }_{\mathcal{K} * \alpha} W_{2}
$$

Arguably, Condition (IndR) is quite natural and not overly constrained: When $\mathcal{K}$ is revised by $\alpha$, Condition (IndR) requires a world $W_{1}$ confirming the new information $\alpha$ to become more plausible than a world $W_{2}$ violating $\alpha$, provided that $W_{1}$ was at least as plausible as $W_{2}$. 


\subsection{Cut Base Revision}

The constructive models based on epistemic entrenchment and/or faithful ranking seem mathematically elegant. However, in order to construct an implementable revision operator, we need a feasible representation of belief states. Obviously, it is infeasible to encode on a computer an explicit belief set, since it is infinite in general. Many researchers |Wobcke, 1992, Nebel, 1994| have therefore suggested to represent the logical contents of a belief state by a belief base, which is a finite set of sentences (not logically closed). Moreover, [Nebel, 1998] has argued that the size of the extra-logical preference information of a belief state should be polynomially bounded by the size of its belief base.

Cut base revision is a revision operator proposed by [Nebel, 1991] based on a very compact belief representation. Formally, a prioritized base $\left\langle B, \leq_{B}\right\rangle$ consists of a belief base $B$ and a total pre-order $\leq_{B}$ on $B$. Note that a prioritized base $\left\langle B, \leq_{B}\right\rangle$ can also be represented by a totally ordered family of sets of sentences $\left(B_{1}, \ldots, B_{n}\right)$ such that $\alpha \leq_{B} \beta$ iff there exist $i, j$ with $\alpha \in B_{i}, \beta \in B_{j}$, and $i \leq j$.

Given a prioritized base $\left\langle B, \leq_{B}\right\rangle$, the cut-set of a sentence $\alpha$ is defined as follows:

$$
\text { cut }_{<_{B}}(\alpha) \stackrel{\text { def }}{=}\left\{\beta \in B \mid\left\{\gamma \in B \mid \beta \leq_{B} \gamma\right\} \not \forall \alpha\right\}
$$

Put in words, the cut-set of $\alpha$ consists of all sentences in $B_{i} \cup \ldots \cup B_{n}$ such that if the next lower class $B_{i-1}$ is added, then $\alpha$ becomes entailed.

Let $C n(B)$ be the belief set induced from a prioritized base $\left\langle B, \leq_{B}\right\rangle$, then according to [Nebel, 1994] the total pre-order $\leq_{B}$ can be generalized to an epistemic entrenchment $\leq_{C n(B)}$ on $C n(B)$ by letting

$$
\alpha \leq_{C n(B)} \beta \text { iff } c u t_{<_{B}}(\beta) \subseteq \operatorname{cut}_{<_{B}}(\alpha)
$$

Formally, cut base revision is defined as follows:

$$
\left\langle B, \leq_{B}\right\rangle *_{\text {cut }} \alpha \stackrel{\text { def }}{=} \operatorname{cut}_{<_{B}}(\neg \alpha) \cup\{\alpha\}
$$

Note that $\mathrm{cut}_{<_{B}}(\neg \alpha)$ is a subset of $B$; therefore, the size of $\left\langle B, \leq_{B}\right\rangle *_{\text {cut }} \alpha$ is linearly bounded in the size of $B$ and $\alpha$.

In [Nebel, 1994] it has been shown that cut base revision is essentially an epistemic entrenchment-based revision: Suppose that $\left\langle B, \leq_{B}\right\rangle$ is a prioritized base and $\leq_{C n(B)}$ is the epistemic entrenchment as defined by (2), then for any sentence $\alpha$ :

$$
C n\left(\left\langle B, \leq_{B}\right\rangle *_{\text {cut }} \alpha\right)= \begin{cases}\mathcal{L} & \text { if } \vdash \neg \alpha \\ \left\{\beta \in \mathcal{L} \mid \neg \alpha<_{C n(B)} \neg \alpha \vee \beta\right\} & \text { otherwise }\end{cases}
$$

Nebel's proposal constitutes a nice step from theory to computation. Unfortunately, cut base revision is not an iterated revision operator since it maps a prioritized base and the new information to a flat belief base. It is therefore impossible to do a subsequent revision. This is also referred to by [Hansson, 2003] as the problem of categorial mis-matching. It is not difficult to see that categorial mis-matching also occurs in the constructive model based on faithful ranking.

\section{Reinforcement Belief Revision}

It is an interesting question whether we can construct a satisfactory iterated revision operator by generalizing cut base revision. Unfortunately, the following discussion will 


\begin{tabular}{|c|}
\hline$B_{n}$ \\
\hline$\vdots$ \\
\hline$B_{i}$ \\
\hline$\alpha$ \\
\hline
\end{tabular}

(a)

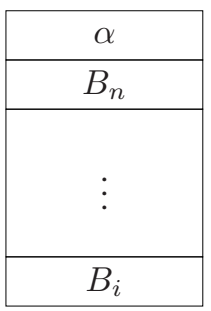

(b)

Figure 1: Two naive generalizations of cut base revision

show that at least a naive approach does not work. To make our presentation easier, we assume that a prioritized base is represented by a totally ordered family of classes of sentences $\left(B_{1}, \ldots, B_{n}\right)$.

Suppose that a prioritized base $\left(B_{1}, \ldots, B_{n}\right)$ is revised by $\alpha$ and cut $_{<_{B}}(\neg \alpha)=$ $\bigcup\left\{B_{i}, \ldots, B_{n}\right\}$. According to 3 , the revised belief base consists of $\left(B_{i}, \ldots, B_{n}\right)$ and $\alpha$. Arguably, we have every reason to assume that $\left(B_{i}, \ldots, B_{n}\right)$ is ordered as before. The only problem is, where to put the new information $\alpha$ ? Without any further information, we may have two options: to render $\alpha$ less plausible than $\left(B_{i}, \ldots, B_{n}\right)$ as shown in Figure 1 a); or to consider $\alpha$ to be more plausible than $\left(B_{i}, \ldots, B_{n}\right)$ as shown in Figure 1 (b).

Formally, we can define the so-called skeptical cut base revision as follows:

$$
\left\langle B, \leq_{B}\right\rangle *_{\text {cut }}^{s} \alpha \stackrel{\text { def }}{=}\left\langle B_{1}=\text { cut }_{<_{B}}(\neg \alpha) \cup\{\alpha\}, \leq_{B_{1}}\right\rangle
$$

where $\beta \leq_{B_{1}} \gamma$ iff $\beta=\alpha$ or $\beta, \gamma \in$ cut $_{<_{B}}(\neg \alpha)$ and $\beta \leq_{B} \gamma$. It is not difficult to see that skeptical cut base revision satisfies the AGM postulates as well as Postulate $(\mathrm{CB})$.

Similarly, the so-called credulous cut base revision is defined as follows:

$$
\left\langle B, \leq_{B}\right\rangle *_{c u t}^{c} \alpha \stackrel{\text { def }}{=}\left\langle B_{1}=\text { cut }_{<_{B}}(\neg \alpha) \cup\{\alpha\}, \leq_{B_{1}}\right\rangle
$$

where $\beta \leq_{B_{1}} \gamma$ iff $\gamma=\alpha$ or $\beta, \gamma \in$ cut $_{<_{B}}(\neg \alpha)$ and $\beta \leq_{B} \gamma$. Credulous cut base revision satisfies the AGM postulates, (DP1), and (Rec), but violates (DP2).

Note that both skeptical and credulous cut base revision have their own assumptions about the plausibility of the new information. One may argue that these assumptions could be violated in many situations. In the sequel, we will present a generalization of cut base revision which deals with the plausibility of the new information in a more appropriate manner.

In our approach, a belief state is represented by a finite set of integer-weighted sentences. Formally, an epistemic entrenchment base (EE base, for short), denoted by $\Xi=\langle B, f\rangle$, consists of a belief base $B$ and a mapping $f$ from $B$ to $\mathbb{N}^{+} 2$ The

\footnotetext{
${ }^{2}$ EE bases and related notions that will be introduced in this section are not new. Similar belief representations have been used in various computational revision operators Williams, 1992 Dixon, 1994 Williams, 1994. Moreover, knowledge bases in possibilistic logic Dubois et al., 1994 are essentially EE bases modulo a monotonic scale transformation. What makes our work distinct, however, is a novel and better approach to revising EE bases. As we will see in Section 4.2 this also gives us a new approach to revising possibilistic knowledge bases.
} 
belief set of $\Xi$ consists of all logical consequences of $B$, that is, $\operatorname{Bel}(\Xi)=C n(B)$. For any sentence $\beta \in B$, we call $f(\beta)$ its evidence degree. Intuitively, a sentence with a larger evidence degree is considered more plausible.

Given an EE base $\Xi=\langle B, f\rangle$, we denote by $\left.\Xi\right|_{m}$ the set of sentences in $B$ whose evidence degree is exactly $m$ :

$$
\left.\Xi\right|_{m} \stackrel{\text { def }}{=}\{\beta \in B \mid f(\beta)=m\}
$$

Moreover, $\Xi^{m}$ is the set of sentences whose evidence degree is greater than $m$ :

$$
\Xi^{m} \stackrel{\text { def }}{=} \bigcup\left\{\left.\Xi\right|_{i} \mid i \geq m\right\}
$$

The belief degree (also called rank) of a sentence $\beta$ wrt. an EE base $\Xi=\langle B, f\rangle$ is defined as follows:

$$
\operatorname{Rank}_{\Xi}(\beta) \stackrel{\text { def }}{=} \begin{cases}0 & \text { if } B \forall \beta \\ \infty & \text { else if } \vdash \beta \\ \max \left(\left\{m \mid \Xi^{m} \vdash \beta\right\}\right) & \text { otherwise }\end{cases}
$$

We may consider an EE base as a collection of some uncertain information, in which the rank of a sentence can be interpreted as its certainty. Note that it is possible that $\operatorname{Rank}_{\Xi}(\beta)>f(\beta)$ for a sentence $\beta \in B$, in which case we say that $\beta$ is redundant in $\Xi$. Therefore, the evidence degree $f(\beta)$ of a sentence $\beta \in B$ is only a lower bound of its belief degree. It is not difficult to see that we can remove redundant sentences from an EE base without affecting the belief degrees of all sentences.

An EE base $\Xi=\langle B, f\rangle$ is a generalized prioritized base in which the cut-set of a sentence $\alpha$ can be obtained as follows:

$$
\operatorname{cut}_{\Xi}(\alpha)=\left\{\beta \in B \mid \operatorname{Rank}_{\Xi}(\alpha)<f(\beta)\right\}
$$

Note that the notion of cut-set defined by (5) generalizes the one defined by 11 .

Not surprisingly, we can also derive an epistemic entrenchment $\leq_{B e l(\Xi)}$ from an EE base $\Xi=\langle B, f\rangle$ by stipulating:

$$
\alpha \leq_{B e l(\equiv)} \beta \text { iff } \operatorname{Rank}_{\Xi}(\alpha) \leq \operatorname{Rank}_{\Xi}(\beta)
$$

In our setting, an iterated revision operator should now be a function which maps an $\mathrm{EE}$ base and the new information to a revised EE base. It is worth mentioning that the quantitative nature of EE bases allows to represent more fine-grained beliefs, e.g., the belief that $\alpha$ is "much more plausible" than $\beta$. As we will see, the quantitative nature of EE bases also allows for a fine-grained control on belief revision. The discussion in Section 2.3 suggests that one major problem is to find an appropriate evidence degree for the new information in the revised EE base. Obviously, if the new information is a mere sentence $\alpha$, then the revision operator has to assign to $\alpha$ an evidence degree via a fixed scheme. But it is unlikely that there exists such a fixed scheme suitable for all different kinds of applications. Therefore, based on the same considerations as in [Spohn, 1988], we consider a more general revision scheme in which the new information consists of a sentence $\alpha$ along with an evidence degree $m \in \mathbb{N}^{+}$.

By generalizing cut base revision, we now define so-called reinforcement base revision as follows:

$$
\begin{aligned}
\langle B, f\rangle *_{r}\langle\alpha, m\rangle \stackrel{\text { def }}{=} & \left\{\left\langle\beta, f(\beta)-\operatorname{Rank}_{\Xi}(\neg \alpha)\right\rangle \mid \beta \in \operatorname{cut} \Xi(\neg \alpha)\right\} \\
& \cup\{\langle\alpha \vee \beta, f(\beta)+m\rangle \mid \beta \in B\} \\
& \cup\{\langle\alpha, m\rangle\}
\end{aligned}
$$


Note that the new sentence $\alpha$ is assigned evidence degree $m$ in the revised EE base. The main difference between cut base revision and reinforcement base revision is that the latter adds a disjunction $\beta \vee \alpha$ for every sentence $\beta \in B$. These disjunctions will obviously not affect the logical contents of the revised EE base, but they are necessary to avoid (undesirable) implicit dependencies 3 It is easy to see that the size of $\langle B, f\rangle *_{r}\langle\alpha, m\rangle$ is linearly bounded by the size of $\langle B, f\rangle$ and $\langle\alpha, m\rangle$.

To see how reinforcement base revision works, let us consider a classical example introduced by [Darwiche and Pearl, 1997].

Example 1. We are introduced to a lady $X$ who sounds smart and looks rich, so we believe that $X$ is smart and $X$ is rich. Since we profess of no prejudice, we also maintain that $X$ is smart even if found to be poor and conversely, $X$ is rich even if found to be not smart. Now, we obtain some evidence that $X$ is in fact not smart, we remain of course convinced that $X$ is rich. Still, it would be strange for us to say, "if the evidence turns out false, and $X$ turns out smart after all, we would no longer believe that $X$ is rich". If we currently believe $X$ is smart and rich, then evidence first refuting then supporting that $X$ is smart should not in any way change our opinion about $X$ being rich.

Let $s$ and $r$ represent respectively that $X$ is smart and $X$ is rich. Assume that our initial belief state is encoded by $\Xi=\{\langle r, 1\rangle,\langle s, 1\rangle,\langle r \vee s, 2\rangle\} 4$ and we first learn that $X$ is not smart with evidence degree 2 . Since $\operatorname{Rank}_{\Xi}(\neg \neg s)=1$, according to (7), the revised EE base is $\Xi_{1}=\Xi *_{r}\langle\neg s, 2\rangle=\{\langle r \vee s, 1\rangle,\langle\neg s, 2\rangle,\langle r \vee \neg s, 3\rangle\} 5$ Suppose we now learn that $X$ is smart with evidence 2 . Since $\operatorname{Rank}_{\Xi_{1}}(\neg s)=2$, we obtain $\Xi_{2}=\Xi_{1} *_{r}\langle s, 2\rangle=\{\langle r \vee \neg s, 1\rangle,\langle s, 2\rangle,\langle r \vee s \vee s, 3\rangle\}$. It is not difficult to see that $\operatorname{Rank}_{\Xi_{1}}(r)=1$, which means that we continue to believe that $X$ is rich (as expected).

As reinforcement base revision is a generalization of cut base revision, one may wonder whether it suffers from a well-known weakness of the latter, namely, the failure of proper belief preservation. Consider the following example 6 Suppose that $\Xi=\{\langle p, 1\rangle,\langle q, 2\rangle,\langle r, 3\rangle\}$ is revised by $\langle\neg q, 4\rangle$. At first glance, it seems indeed undesirable that, in the revised EE base $\{\langle r, 1\rangle,\langle p \vee \neg q, 5\rangle,\langle r \vee \neg q, 7\rangle,\langle\neg q, 4\rangle\}, p$ is not believed anymore and the rank of $r$ is lowered to 1 . However, this is indeed the intended behavior, as the following argument shows.

As we have noted in [Jin and Thielscher, 2007], for any revision operator satisfying the AGM postulates a belief state (regardless of its representation) will encode some implicit dependencies among beliefs. More specifically, Condition $(C *)$ in Section 2.1.1 shows that if $\beta \vee \neg \alpha$ is not more entrenched than $\neg \alpha$, then $\beta$ will not survive an $\alpha$-revision (because $\beta$ depends on $\neg \alpha$, so its removed together with $\neg \alpha$ ). In the above case, $\Xi$ encodes implicitly that " $p$ depends on $q$ " since $p \vee q$ is not considered to be more plausible than $q$. Therefore, it is perfectly reasonable and inevitable that $p$ is removed after a revision by $\neg q$. An interesting question is how we can obtain a belief state which does not encode undesirable implicit dependencies like " $p$ depends on $q$ "? In fact, it is a major advantage of reinforcement base revision that

\footnotetext{
${ }^{3}$ We refer readers who are interested in the problem of implicit dependencies to [Jin and Thielscher, 2007 for a detailed discussion.

${ }^{4}$ Readers are invited to check that $\Xi$ is equal to $\left(\emptyset *_{r}\langle r, 1\rangle\right) *_{r}\langle s, 1\rangle$ as well as $\left(\emptyset *_{r}\langle s, 1\rangle\right) *_{r}\langle r, 1\rangle$.

${ }^{5}$ For simplicity, redundant sentences have been removed, and we will always tacitly do so in the rest of the paper.

${ }^{6}$ We are grateful to an anonymous reviewer of this paper for suggesting this example.
} 
it will not introduce undesirable dependencies. For instance, assume that the initial belief state is empty and we subsequently learn $p, q, r$ with individual evidence degrees. In this case, $\Xi^{\prime}=\left(\left(\emptyset *_{r}\langle p, 1\rangle\right) *_{r}\langle q, 2\rangle\right) *_{r}\langle r, 3\rangle$ will encode implicitly that $p, q, r$ are independent of each other. Therefore, a revision by the negation of one proposition will not alter the ranks of any of the other propositions. For example, it is not difficult to see that $\operatorname{Rank}_{\Xi^{\prime \prime}}(p)=1$ and $\operatorname{Rank}_{\Xi^{\prime \prime}}(r)=3$, where $\Xi^{\prime \prime}=\Xi^{\prime} *_{r}\langle\neg q, 4\rangle$.

The fact that $r$ is lowered to 1 in the first example, $\{\langle p, 1\rangle,\langle q, 2\rangle,\langle r, 3\rangle\} *_{r}$ $\langle\neg q, 4\rangle$, can be explained as follows. Since $q$ depends on $r$ in the initial belief state, $r$ can be considered a strong support of $q$. Therefore, it is reasonable that we become less certain about $r$ after $q$ is learned to be false. In contrast, the rank of $r$ will remain the same when $\Xi^{\prime}$ is revised by $\neg q$ because $q$ is not dependent on $r$ in $\Xi^{\prime}$. The following result shows that a revision of one sentence in general will not affect the rank of another sentence, unless they are somehow logically related:

Proposition 3.1. Suppose that $\Xi_{1}$ is the result of revising an EE base $\Xi$ by $\langle\neg \alpha, m\rangle$, where $\alpha$ is an arbitrary sentence and $m$ an arbitrary natural number. Then for any sentence $\beta$ :

$$
\operatorname{Rank}_{\Xi_{1}}(\beta) \neq \operatorname{Rank}_{\Xi}(\beta) \text { iff } \operatorname{Rank}_{\Xi_{2}}(\alpha) \neq \operatorname{Rank}_{\Xi}(\alpha)
$$

where $\Xi_{2}=\Xi *_{r}\left\langle\neg \beta, m^{\prime}\right\rangle$ for an arbitrary natural number $m^{\prime}$.

We remark that more logical properties of reinforcement base revision will be presented later in this section.

\subsection{OCF-Based Semantics}

To provide a better intuition about reinforcement base revision, we give an alternative, semantic characterization, which originates in [Jin and Thielscher, 2007] and is based on Spohn's ordinal conditional functions (OCF, for short) [Spohn, 1988]. Originally, an OCF $k$ is a mapping from the set of all possible worlds $\Theta_{\mathcal{L}}$ to the class of ordinals. Like in |Spohn, 1991|, for mathematical simplicity we take the range of an OCF $k$ to be the natural numbers, $\mathbb{N}$. For any possible world $W$, we call $k(W)$ the rank of $W$. Intuitively, the rank of a world represents its degree of implausibility. Hence, the lower its rank, the more plausible is a world.

Any OCF $k$ is a belief state from which we can induce a belief set and a faithful ranking $\preceq_{k}$, where $\operatorname{Bel}(k)$ is the set of sentences which hold in all worlds of rank 0 :

$$
\operatorname{Bel}(k)=\operatorname{Th}(\{W \mid k(W)=0\})
$$

In contrast to [Spohn, 1988], we do not require the set of possible worlds with rank 0 to be non-empty. Therefore, our approach can also deal with inconsistent belief sets.

An OCF $k$ is extended to a ranking of sentences as follows:

$$
k(\beta)= \begin{cases}\infty & \text { if } \vdash \beta \\ \min \{k(W) \mid W \models \neg \beta)\} & \text { otherwise }\end{cases}
$$

Put in words, the rank of a sentence is the lowest rank of a world in which the sentence does not hold 7 Hence, the higher the rank of a sentence, the firmer the belief in it, and the belief set consists of all sentences with rank greater than 0 .

\footnotetext{
${ }^{7}$ In Spohn's original proposal, the rank of a sentence is the lowest rank of a world in which it is true. So the rank of $\beta$ there is equal to $k(\neg \beta)$ here.
} 


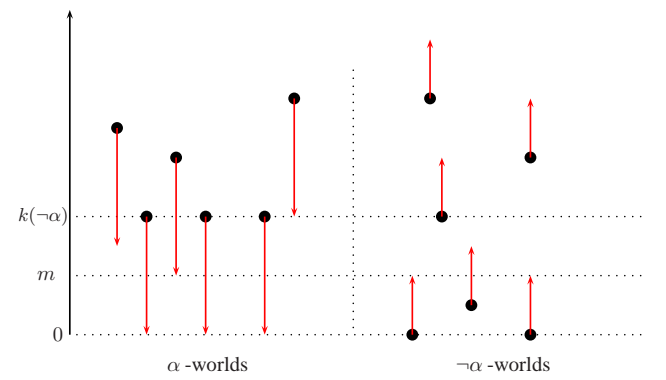

Figure 2: Visualization of reinforcement OCF revision

It is not difficult to see that an OCF $k$ determines an epistemic entrenchment as follows:

$$
\alpha \leq_{k} \beta \text { iff } k(\alpha) \leq k(\beta)
$$

In the sequel, we present a generalized revision operator (called reinforcement $O C F$ revision) which also allows to assign different evidence degrees to new information. An OCF $k$ is revised according to new information $\alpha$ with evidence degree $m \in \mathbb{N}^{+}$as follows:

$$
\left(k_{\alpha . m}^{r, *}\right)(W) \stackrel{\text { def }}{=} \begin{cases}k(W)-k(\neg \alpha) & \text { if } W \models \alpha \\ k(W)+m & \text { otherwise }\end{cases}
$$

Put in words, the rank of an $\alpha$-world decreases by $k(\neg \alpha)$, whereas the rank of a $\neg \alpha$-world increases by $m$.

A graphical illustration of the principle of reinforcement OCF revision is given in Figure 2 Circles to the left (right) of the vertical dotted line represent $\alpha$-worlds ( $\neg \alpha$-worlds, respectively); the vertical coordinate of a possible world denotes its rank; and the arrow starting from a possible world indicates the change of its rank.

\subsubsection{Equivalence Results}

To show the equivalence of reinforcement belief revision and reinforcement OCF revision, we need a mapping from EE bases to OCFs. Formally, an OCF $k_{\Xi}$ is derived from an EE base $\Xi=\langle B, f\rangle$ by letting

$$
k_{\Xi}(W)= \begin{cases}0 & \text { if } W \models B \\ \max (\{f(\beta) \mid \beta \in B \text { and } W \not \models \beta\}) & \text { otherwise }\end{cases}
$$

Put in words, the rank of a possible world is the maximal evidence degree of all sentences that it does not satisfy.

The following result shows that an EE base and its induced OCF encode essentially the same belief state 8

Proposition 3.2. Suppose that $\Xi=\langle B, f\rangle$ is an EE base and $k_{\Xi}$ the induced OCF as defined by [12], then for any sentence $\beta$ :

$$
\operatorname{Rank}_{\Xi}(\beta)=k_{\Xi}(\beta)
$$

${ }^{8} \mathrm{~A}$ similar result can be found in possiblistic logic Dubois et al., 1994 (cf. Proposition 4.3. 


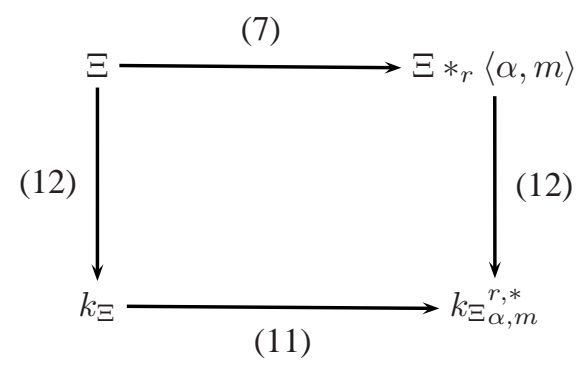

Figure 3: A commuting diagram for reinforcement base revision and reinforcement OCF revision

Recall Example 1] It is easy to see that the EE base $\Xi=\{\langle r, 1\rangle,\langle s, 1\rangle,\langle r \vee s, 2\rangle\}$ induces the OCF $k$ shown in Table 1 (middle column; assuming $\mathcal{P}=\{s, t\}$ ). Now, with $k(\neg \neg s)=1$ and following (11) we obtain the OCF $k_{\neg s, 2}^{r, *}$ shown in the right column of Table 1 We remark that this $k_{\neg s, 2}^{r, *}$ is exactly the OCF induced from the EE base $\Xi_{1}=\{\langle r \vee s, 1\rangle,\langle\neg s, 2\rangle,\langle r \vee \neg s, 3\rangle\}$, which is the result of revising $\Xi$ by $\neg s$ with evidence degree 2 .

\begin{tabular}{|l|c|c|}
\hline possible worlds & $k$ & $k_{\neg s, 2}^{r, *}$ \\
\hline \hline$W_{1}=\{s, r\}$ & 0 & 2 \\
$W_{2}=\{s\}$ & 1 & 3 \\
$W_{3}=\{r\}$ & 1 & 0 \\
$W_{4}=\{\}$ & 2 & 1 \\
\hline
\end{tabular}

Table 1: An example of reinforcement OCF revision

The following theorem shows that the two revision operators behave symmetrically in general. That is to say, for any EE base $\Xi$ and new information $\langle\alpha, m\rangle$, the revised EE base $\Xi *_{r}\langle\alpha, m\rangle$ induces exactly the revised OCF $k_{\Xi_{\alpha, m}^{r, *}}^{r \text { (see also Figure 3. }}$.

Theorem 3.3. Suppose that $\Xi$ is an EE base and $k_{\Xi}$ the OCF induced from $\Xi$. Let $\langle\alpha, m\rangle$ be any new information, then for all possible worlds $W$ :

$$
k_{\Xi_{1}}(W)=k_{\Xi_{\alpha, m}}^{r, *}(W)
$$

where $\Xi_{1}=\Xi *_{r}\langle\alpha, m\rangle$.

As a direct consequence of Proposition 3.2 and Theorem 3.3 we have the following equivalence result.

Corollary 3.4. Suppose that $\Xi$ is an EE base and $k_{\Xi}$ the OCF induced from $\Xi$. Let $\langle\alpha, m\rangle$ be any new information, then for any sentence $\beta$ :

$$
\operatorname{Rank}_{\Xi_{1}}(\beta)=k_{\Xi_{\alpha, m}}^{r, *}(\beta)
$$

where $\Xi_{1}=\Xi *_{r}\langle\alpha, m\rangle$. 


\subsection{Logical Properties}

The equivalence result enables us to prove important logical properties of reinforcement base revision by referring to reinforcement OCF revision instead. The reason for doing so is two-fold: On the one hand, it is much easier to deal with the latter due to its simple and intuitive construction. Moreover, reinforcement OCF revision has already been investigated in [Jin and Thielscher, 2007]. In the following, we summarize the most important of the known properties.

Theorem 3.5. |Jin and Thielscher, 2007| Consider an OCF $k$.

1. Assume an arbitrary but fixed evidence degree for any new information. Then reinforcement $O C F$ revision satisfies the $A G M$ postulates $(\mathcal{K} * 1)-(\mathcal{K} * 8)$ as well as (DP1), (DP2), and (Ind).

2. For any $m_{1}, m_{2} \in \mathbb{N}^{+}$, reinforcement OCF revision satisfies the following:

$$
\begin{aligned}
& \text { (EDP1) If } \beta \vdash \alpha \text {, then }\left(k_{\alpha, m_{1}}^{r, *}\right)_{\beta, m_{2}}^{r, *} \equiv k_{\beta, m_{2}}^{r, *} \\
& \text { (EDP2) } \quad \text { If } \beta \vdash \neg \alpha \text {, then }\left(k_{\alpha, m_{1}}^{r, *}\right)_{\beta, m_{2}}^{r, *} \equiv k_{\beta, m_{2}}^{r, *} \\
& \text { (EInd) } \quad \text { If there exists } m \text { such that } k_{\neg \beta, m_{2}}^{r, *} \nvdash \neg \alpha \text {, then } \\
& \quad\left(k_{\alpha, m_{1}}^{r, *}\right)_{\neg \beta, m_{2}}^{r, *} \vdash \alpha
\end{aligned}
$$

3. Let $\langle\alpha, m\rangle$ be some new information, then

$$
k_{\alpha, m}^{r, *}(\alpha)=k(\alpha)+m
$$

All of these formal properties of reinforcement OCF revision are inherited by reinforcement base revision. Specifically,

1. Assuming an arbitrary but fixed evidence degree for any new information, we obtain a standard iterated revision operator and the desirable rationality postulates are satisfied;

2. This holds also for the more general case of varying evidence degrees;

3. Reinforcement base revision does indeed have a reinforcement effect, which, from a pragmatic point of view, is a desirable effect in particular for domains with several independent information sources.

This is summarized in the following corollary, which is a direct consequence of the above-mentioned properties and the equivalence of reinforcement belief revision and reinforcement base revision.

Corollary 3.6. Consider an EE-base $\Xi$.

1. Assume an arbitrary but fixed evidence degree for any new information. Then reinforcement base revision satisfies the AGM postulates $(\mathcal{K} * 1)-(\mathcal{K} * 8)$ as well as (DP1), (DP2), and (Ind).

2. For any $m_{1}, m_{2} \in \mathbb{N}^{+}$, reinforcement base revision satisfies the following:

$$
\begin{array}{ll}
\left(E D P 1^{\prime}\right) & \text { If } \beta \vdash \alpha \text {, then }\left(\Xi *_{r}\left\langle\alpha, m_{1}\right\rangle\right) *_{r}\left\langle\beta, m_{2}\right\rangle \equiv \Xi *_{r}\left\langle\beta, m_{2}\right\rangle \\
\left(E D P 2^{\prime}\right) & \text { If } \beta \vdash \neg \alpha \text {, then }\left(\Xi *_{r}\left\langle\alpha, m_{1}\right\rangle\right) *_{r}\left\langle\beta, m_{2}\right\rangle \equiv \Xi *_{r}\left\langle\beta, m_{2}\right\rangle
\end{array}
$$


(EInd') If there exists $m$ such that $\Xi *_{r}\langle\beta, m\rangle \nvdash \neg \alpha$, then

$$
\left(\Xi *_{r}\left\langle\alpha, m_{1}\right\rangle\right) *_{r}\left\langle\beta, m_{2}\right\rangle \vdash \alpha
$$

3. Let $\langle\alpha, m\rangle$ be some new, non-tautological information. Let $\Xi_{1}=\Xi *_{r}\langle\alpha, m\rangle$, then

$$
\operatorname{Rank}_{\Xi_{1}}(\alpha)=\operatorname{Rank}_{\Xi}(B, \alpha)+m
$$

\subsubsection{Degree of Syntax Irrelevance}

Strictly speaking, reinforcement base revision violates Dalal's principle of irrelevance of syntax [Dalal, 1988], because the revised belief state is not purely determined by the logical contents of the original belief state. The principle of irrelevance of syntax is motivated by Newell's influential proposal which states that the behavior of an intelligent system should be specifiable on the knowledge level |Newell, 1982|; that is to say, the behavior of the system is determined solely by the contents of its knowledge (in our context, beliefs), independent of the symbolic representation. In the following, we show that reinforcement base revision does indeed not depend on the syntax of an EE base, so that it actually complies with Newell's idea.

To this end, we call two EE bases $\Xi_{1}$ and $\Xi_{2}$ epistemically equivalent iff their induced epistemic entrenchments (as defined by (6) are equivalent; that is, for any sentences $\alpha, \beta$ :

$$
\alpha \leq_{\operatorname{Bel}\left(\Xi_{1}\right)} \beta \text { iff } \alpha \leq_{\operatorname{Bel}\left(\Xi_{2}\right)} \beta
$$

The following result shows that two epistemically equivalent EE bases yield the same beliefs when revised by the same sentence.

Proposition 3.7. Let $\Xi_{1}, \Xi_{2}$ be two epistemically equivalent EE bases, then for any sentence $\alpha$ and evidence degrees $m_{1}, m_{2} \in \mathbb{N}^{+}$:

$$
\operatorname{Bel}\left(\Xi_{1}^{\prime}\right)=\operatorname{Bel}\left(\Xi_{2}^{\prime}\right)
$$

where $\Xi_{1}^{\prime}=\Xi_{1} *_{r}\left\langle\alpha, m_{1}\right\rangle$ and $\Xi_{2}^{\prime}=\Xi_{2} *_{r}\left\langle\alpha, m_{2}\right\rangle$.

Moreover, we call two EE bases $\Xi_{1}$ and $\Xi_{2}$ equivalent, denoted by $\Xi_{1} \cong \Xi_{2}$, iff for any sentence $\beta$ :

$$
\operatorname{Rank}_{\Xi_{1}}(\beta)=\operatorname{Rank}_{\Xi_{2}}(\beta)
$$

Obviously, two equivalent EE bases are also epistemically equivalent, which in turn means that they are logically equivalent.

It is not difficult to see that two equivalent $\mathrm{EE}$ bases lead to equivalent $\mathrm{EE}$ bases when revised by the same information.

Proposition 3.8. Let $\Xi_{1}, \Xi_{2}$ be two equivalent EE bases and $\langle\alpha, m\rangle$ any new information, then

$$
\Xi_{1} *_{r}\langle\alpha, m\rangle \cong \Xi_{2} *_{r}\langle\alpha, m\rangle
$$

\subsection{Computational Complexity}

In practice, computational complexity is a very important criterion for evaluating revision operators [Eiter and Gottlob, 1992, Liberatore, 1997b, Cadoli et al., 1995| 9 Like in |Eiter and Gottlob, 1992], we will consider the so-called problem of counterfactual

\footnotetext{
${ }^{9}$ We assume the reader to have basic knowledge in complexity theory, as can be found, e.g., in [Papadimitriou, 1994.
} 
(CF, for short), which means to decide whether $\mathcal{K} * \alpha \vdash \beta$ holds for arbitrary belief states $\mathcal{K}$ and sentences $\alpha, \beta$.

[Nebel, 1992] has shown that both SAT and VALID can be polynomially (manyto-one) reduced to the problem of $\mathrm{CF}$ for a revision operator that satisfies $(\mathcal{K} * 4)$ and $(\mathcal{K} * 5)$. This means that the problem of $\mathrm{CF}$ is at least both NP-hard and coNP-hard. It follows immediately that in general the complexity of the problem of CF is higher than $\mathrm{NP}$ and coNP, provided that NP $\neq$ coNP.

For most well-known belief change operators in the literature, the problem of $\mathrm{CF}$ has been shown to be at the lower end of so-called polynomial hierarchy |Eiter and Gottlob, 1992, Nebel, 1994, Liberatore, 1997al. In the following, we give a brief introduction to this concept. Let $X$ be a class of decision problems. Let $\mathrm{P}^{X}$ denote the class of decision problems that can be decided in polynomial time by a deterministic Turing machine that is allowed to use a procedure (also referred to as an oracle) for deciding a problem $Q \in X$, whereby executing the procedure only costs constant time. Similarly, $\mathrm{NP}^{X}$ denotes the class of decision problems that can be decided in polynomial time by a non-deterministic Turing machine that is allowed to use an oracle for deciding a problem $Q \in X$. Based on these notions, the complexity classes $\Delta_{k}^{p}, \Sigma_{k}^{p}$, and $\Pi_{k}^{p}$ are inductively defined for $k=0,1,2, \ldots$ :

$$
\begin{aligned}
& \Delta_{0}^{p}=\Sigma_{0}^{p}=\Pi_{0}^{p}=\mathrm{P} \\
& \Delta_{k+1}^{p}=\mathrm{P}^{\Sigma_{k}^{p}} \\
& \Sigma_{k+1}^{p}=\mathrm{NP}^{\Sigma_{k}^{p}} \\
& \Pi_{k}^{p}=\operatorname{co} \Sigma_{k+1}^{p}
\end{aligned}
$$

Note that $\Sigma_{1}^{p}=\mathrm{NP}$ and $\Pi_{1}^{p}=$ coNP. The polynomial hierarchy is then defined as $\mathrm{PH}=\bigcup_{k \geq 0} \Delta_{k}^{p}=\bigcup_{k \geq 0} \Sigma_{k}^{p}=\bigcup_{k \geq 0} \Pi_{k}^{p} \subseteq$ PSPACE. It is unknown, but commonly believed, that the inclusion between $\mathrm{PH}$ and PSPACE is a proper one.

For problems in $\Delta_{2}^{p}$, it is often difficult to determine their exact complexity. But by restricting the number of oracle calls, we obtain an important special class $\Delta_{2}^{p}[\mathrm{O}(\log n)]$ of problems that can be decided in polynomial time by using only logarithmically many oracle calls. Furthermore, inside of $\Delta_{2}^{p}[\mathrm{O}(\log n)]$ are the classes of the so-called boolean hierarchy, that is, problems solvable in deterministic polynomial time using a constant number of NP-oracle calls.

It has been shown in INebel, 1994 that for cut base revision the problem of CF is $\Delta_{2}^{\mathrm{p}}[\mathrm{O}(\log \mathrm{n})]$-complete. Since reinforcement base revision generalizes cut base revision, it does not come as a surprise that one can show that the former has the same complexity:

Theorem 3.9. For reinforcement base revision, the problem of $C F$ is $\Delta_{2}^{\mathrm{p}}[\mathrm{O}(\log \mathrm{n})]$ complete.

This theorem shows that there is not too much room for improvement in terms of computational complexity, since $\Delta_{2}^{\mathrm{p}}[\mathrm{O}(\log \mathrm{n})]$ inhabits the very low end of the polynomial hierarchy. In general, unless the size of a belief state is restricted, it is hard to imagine that the problem of $\mathrm{CF}$ for any realistic operator will only require a constant number of NP-oracle calls. Of course, if the underlying language is constrained to Horn sentences, then the problem of CF for reinforcement base revision becomes tractable (i.e., solvable in polynomial time).

It is also an interesting problem to ask how hard it is to compute the revised belief state given an arbitrary belief state and new information. This is the so-called function problem, which is not a decision problem like the problem of $\mathrm{CF}$. The complexity 
classes for decision problems have natural counterparts for function problems: For example, $\mathrm{FP}^{\mathrm{NP}}$ (also referred to as NP -easy) represents the set of all function problems which can be solved in polynomial time by a deterministic Turing machine that is allowed to invoke an NP -oracle. It turns out that the problem of computing a revised belief state (EE base) for reinforcement base revision falls in an interesting complexity class, called NP-equivalent. Formally, a function problem is NP-equivalent iff it is both NP -easy and NP-hard. Note that NP-equivalent is the analogue of NP complete for function problems, in the sense that if one NP-equivalent problem can be solved in polynomial time, then so can be all other NP -equivalent problems.

Theorem 3.10. For reinforcement base revision, the problem of computing a revised belief state is $\mathrm{NP}$-equivalent.

\section{Possibilistic Reinforcement Revision}

Possibility theory (also referred to as possibilistic logic) is another research field which is intimately related to belief revision [Dubois and Prade, 1991, Dubois et al., 1994]. The main difference is that in the former the certainty of information is represented numerically, whereas in the latter we use ordinal measurements [Dubois et al., 1998]. Therefore, many notions used in Section 3 can also be found in possibility theory modulo an innocuous affine transformation. In [Benferhat et al., 2002], for instance, it has been shown that some well-known OCF revision operators can be directly mapped to conditioning operations in possibility theory. In this section, we will likewise show how our reinforcement belief revision can be re-cast in the possibilistic setting. The result is a novel approach to revising possibilistic knowledge states.

In possibility theory, the beliefs of an agent are represented by a possibility distribution $\pi$, which maps the set of all possible worlds $\Theta_{\mathcal{L}}$ to the unit interval $[0,1]$. Contrary to an OCF, a possibility distribution $\pi$ assigns higher possibility to more plausible worlds, so that $\pi(W)=0$ indicates an impossible world $W$ while $\pi(W)=1$ means that nothing prevents $W$ from being the real world.

The belief set $\operatorname{Bel}(\pi)$ encoded by a possibility distribution $\pi$ consists of all sentences which are true in all most plausible worlds:

$$
\operatorname{Bel}(\pi)=\operatorname{Th}(\{W \mid \pi(W)=1\})
$$

Given a possibility distribution $\pi$, we can define two different measures for a sentence $\beta$ : The possibility degree $\Pi_{\pi}(\beta)$ evaluates the extent to which $\beta$ is consistent with $\pi$ :

$$
\Pi_{\pi}(\beta) \stackrel{\text { def }}{=} \begin{cases}0 & \text { if } \vdash \neg \beta \\ \max (\{\pi(W) \mid W \models \beta\}) & \text { otherwise }\end{cases}
$$

The necessity degree $N_{\pi}(\beta)$ evaluates the extent to which $\beta$ is entailed by $\pi 10$

$$
N_{\pi}(\beta) \stackrel{\text { def }}{=} \begin{cases}\infty & \text { if } \vdash \beta \\ 1-\Pi_{\pi}(\neg \beta) & \text { otherwise }\end{cases}
$$

Henceforth, when $\pi$ is obvious from the context, we denote $N_{\pi}(\beta)$ and $\Pi_{\pi}(\beta)$ simply by $N(\beta)$ and $\Pi(\beta)$, respectively.

\footnotetext{
${ }^{10}$ Originally, the necessity degree is simply defined as $N_{\pi}(\beta) \stackrel{\text { def }}{=} 1-\Pi_{\pi}(\neg \beta)$. In this paper, we will use this slightly different definition in order to be fully compatible with the notion of belief degree introduced in Section 3
} 


\subsection{Possibilistic Conditionings}

In possibility theory, the revision of a possibility distribution $\pi$ by a totally reliable evidence $\alpha$ is obtained by Bayesian-style conditioning [Benferhat et al., 2002], that is, for any sentence $\alpha$ and any possible world $W$ :

$$
\pi_{\alpha}^{*}(W)=\pi(W \mid \alpha)
$$

where $\pi(. \mid \alpha)$ is the posterior possibility distribution conditioned on $\alpha$.

Two main methods for conditioning have been proposed in the literature. The socalled minimum-based conditioning is more of a qualitative nature:

$$
\pi\left(\left.W\right|_{m} \alpha\right) \stackrel{\text { def }}{=} \begin{cases}1 & \text { if } \pi(W)=\Pi(\alpha) \text { and } W \models \alpha \\ \pi(W) & \text { else if } \pi(W)<\Pi(\alpha) \text { and } W \models \alpha \\ 0 & \text { otherwise }\end{cases}
$$

Much like genuine Bayesian conditioning, the so-called product-based conditioning re-scales upwards all models of the new evidence $\alpha$ :

$$
\pi\left(\left.W\right|_{\times} \alpha\right) \stackrel{\text { def }}{=} \begin{cases}\frac{\pi(W)}{\Pi(\alpha)} & \text { if } W \models \alpha \text { and } \Pi(\alpha) \neq 0 \\ 1 & \text { else if } W \models \alpha \text { and } \Pi(\alpha)=0 \\ 0 & \text { otherwise }\end{cases}
$$

It is not difficult to see that both $\pi\left(.\left.\right|_{m} \alpha\right)$ and $\pi\left(.\left.\right|_{\times} \alpha\right)$ upgrade the necessity degree of $\alpha$ to 1 , provided that $\nvdash \alpha$. Moreover, they are justified to be called conditioning since both of them satisfy the Bayesian condition $\pi(W)=\pi(W \mid \alpha) \otimes \Pi(\alpha)$ with $\otimes=\min$ (the minimum operator) for minimum-based conditioning and $\otimes=\times$ (the arithmetic product) for product-based conditioning.

When the new evidence $\alpha$ is not fully certain, it may come with its own degree $w \in[0,1]$ of possibility. Based on the well-known Jeffrey Rule [Jeffrey, 1965], a possibility distribution $\pi$ should be conditioned (revised) by an uncertain evidence $\langle\alpha, w\rangle$ as follows:

$$
\pi_{\langle\alpha, w\rangle}^{*}(W) \stackrel{\text { def }}{=} \pi(W \mid\langle\alpha, w\rangle)
$$

where

$$
\pi(W \mid\langle\alpha, w\rangle)= \begin{cases}\pi(W \mid \alpha) & \text { if } W \models \alpha \\ (1-w) \otimes \pi(W \mid \neg \alpha) & \text { otherwise }\end{cases}
$$

Again, $\otimes=\min$ for minimum-based conditioning and $\otimes=\times$ for product-based conditioning. Note that $\pi(. \mid\langle\alpha, w\rangle)$ coincides with $\pi(. \mid \alpha)$ in case $w=1$. Moreover, both minimum-based and product-based conditioning (with uncertain input $\langle\alpha, w\rangle$ ) have the effect that the posterior necessity degree of $\alpha$ is exactly $w$.

In [Benferhat et al., 2002] it has been pointed out that there is an intimate relation between OCF-based revision operators and conditioning in possibility theory. In particular, a scale transformation from an OCF $k$ to a possibility distribution $\pi_{k}$ can be obtained by letting:

$$
\pi_{k}(W)=e^{-k(W)}
$$

Then minimum-based and product-based conditioning with uncertain input can be shown to correspond to two well-known OCF revision operators: adjustment and conditionalization, respectively 11

\footnotetext{
${ }^{11} \mathrm{~A}$ comparison between reinforcement OCF revision and those two operators can be found in Section 5
} 


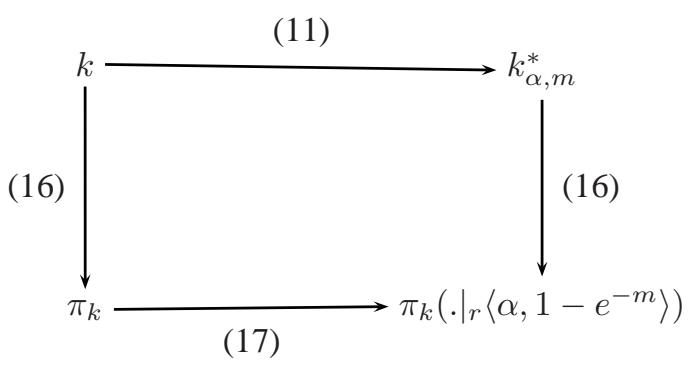

Figure 4: Correspondence between reinforcement OCF revision and conditioning

\subsection{Possibilistic Reinforcement Revision}

We are now ready to present possibilistic versions for both reinforcement OCF and reinforcement base revision. The equivalence between possibilistic reinforcement revision and genuine reinforcement revision will be shown.

\subsubsection{Reinforcement Conditioning}

By slightly modifying product-based conditioning, we obtain the following reinforcement conditioning:

$$
\pi\left(\left.W\right|_{r}\langle\alpha, w\rangle\right)= \begin{cases}\pi\left(\left.W\right|_{\times} \alpha\right) & \text { if } W \models \alpha \\ (1-w) \times \pi(W) & \text { otherwise }\end{cases}
$$

Note that reinforcement conditioning uses $\pi(W)$ to compute the posterior possibility of $\neg \alpha$-worlds, instead of $\pi(W \mid \neg \alpha)$ (as used in product-based conditioning). The main effect of this modification is that the posterior necessity degree of $\alpha$ may not be $w$ anymore. In fact, it can be shown that reinforcement conditioning always strengthens the necessity degree of the new evidence.

Proposition 4.1. Suppose that a possibility distribution $\pi$ is revised by a new nontautological evidence $\alpha$ with possibility degree $w$. Let $\pi_{1}=\pi_{\langle\alpha, w\rangle}^{*}$, then

$$
N_{\pi_{1}}(\alpha)=N_{\pi}(\alpha)+w-w \times N_{\pi}(\alpha)
$$

Since $w \geq w \times N_{\pi}(\alpha)$, this implies that $N_{\pi_{1}}(\alpha) \geq N_{\pi}(\alpha)$. Moreover, it is easy to see that $N_{\pi_{1}}(\alpha)=1$ iff $w=1$ or $N_{\pi}(\alpha)=1$.

We now show that there is actually a close correspondence between reinforcement conditioning and reinforcement OCF revision, given the mapping defined by (16) (cf. also the commuting diagram in Figure 4 .

Theorem 4.2. Suppose that $k$ is an $O C F$ and $\langle\alpha, m\rangle$ (with $m \in \mathbb{N}^{+}$) some input information. Let $k_{\langle\alpha, m\rangle}^{*}$ be the revised OCF using reinforcement OCF revision, then for any possible world $W$ :

$$
\pi_{k_{\langle\alpha, m\rangle}^{*}}(W)=\pi_{k}\left(\left.W\right|_{r}\left\langle\alpha, 1-e^{-m}\right\rangle\right)
$$

The reader may note that an evidence degree $m$ in OCF revision is monotonically mapped to a possibility degree $1-e^{-m}$ in possibilistic conditioning. 


\subsubsection{Reinforcement Possibility Base Revision}

Possibilistic bases provide a method for the compact representation of beliefs in possibility theory. Formally, a possibilistic base $\Sigma$ consists in a belief base $B$ and a mapping $g$ from $B$ to $[0,1]$, where $g(\beta)$ is called the possibility degree of a sentence $\beta \in B$. Intuitively, sentences with higher certainty have a higher possibility degree.

We remark that a possibilistic base is the same as an EE base modulo a monotonic scale transformation. Therefore, many notions on EE bases can also be applied to possibilistic bases. Specifically, given a possibilistic base $\Sigma=\langle B, g\rangle$, let $\Sigma^{w}$ denotes the set of sentences whose possibility degree is at least $w$ :

$$
\Sigma^{w} \stackrel{\text { def }}{=}\left\{\beta_{i} \in B \mid g\left(\beta_{i}\right) \geq w\right\}
$$

Moreover, $\Sigma^{>w}$ is the set of sentences with possibility degree greater than $w$ :

$$
\Sigma^{>w} \stackrel{\text { def }}{=}\left\{\beta_{i} \in B \mid g\left(\beta_{i}\right)>w\right\}
$$

In analogy to the notion of a belief degree, we define the necessity degree of a sentence $\beta$ as follows:

$$
N_{\Sigma}(\beta) \stackrel{\text { def }}{=} \begin{cases}0 & \text { if } B \not \beta \\ \infty & \text { else if } \vdash \beta \\ \max \left(\left\{w \mid \Sigma^{w} \vdash \beta\right\}\right) & \text { otherwise }\end{cases}
$$

Now we are ready to define a revision operator for possibilistic bases. The basic idea is quite similar to that of reinforcement base revision. Suppose that $\Sigma=$ $\left\{\left\langle\beta_{1}, w_{1}\right\rangle, \ldots,\left\langle\beta_{n}, w_{n}\right\rangle\right\}$ is a possibilistic base and $\langle\alpha, w\rangle$ the uncertain new information. Let $\bar{w}=N_{\Sigma}(\neg \alpha)$, then the revised possibilistic base $\Sigma_{1}=\Sigma Q_{r}\langle\alpha, w\rangle$ is obtained as follows:

$$
\begin{aligned}
\Sigma_{1}= & \left\{\left\langle\beta_{i}, \frac{w_{i}-\bar{w}}{1-\bar{w}}\right\rangle \mid w_{i}>\bar{w}\right\} \\
& \cup\{\langle\alpha, w\rangle\} \\
& \cup\left\{\left\langle\alpha \vee \beta_{i}, w_{i}+w-w \times w_{i}\right\}\right.
\end{aligned}
$$

In the sequel, we will show that possibilistic reinforcement base revision is equivalent to reinforcement conditioning. Like in Section 3.1.1 we first define a mapping from a possibilistic base $\Sigma=\left\{\left\langle\beta_{1}, w_{1}\right\rangle, \ldots,\left\langle\beta_{n}, w_{n}\right\rangle\right\}$ to a possibility distribution $\pi_{\Sigma}$ :

$$
\pi_{\Sigma}(W)= \begin{cases}1 & \text { if } W \models\left\{\beta_{i}, \ldots, \beta_{n}\right\} \\ 1-\max \left(\left\{w_{i} \mid W \not \models \beta_{i}\right\}\right) & \text { otherwise }\end{cases}
$$

The following result shows that a possibilistic base $\Sigma$ and its induced possibility distribution $\pi_{\Sigma}$ encode essentially the same belief state.

Proposition 4.3. [Dubois et al., 1994| Suppose that $\Sigma$ is a possibilistic base and $\pi_{\Sigma}$ the induced possibility distribution as defined by (20), then for any sentence $\beta$ :

$$
N_{\Sigma}(\beta)=N_{\pi_{\Sigma}}(\beta)
$$

Finally, we can show the equivalence of reinforcement possibilistic base revision and reinforcement conditioning (cf. Figure 5. 


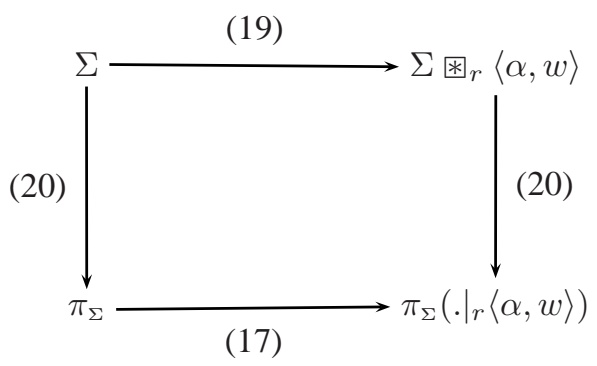

Figure 5: Reinforcement conditioning and possibilistic base revision

Theorem 4.4. Suppose that $\Sigma$ is a possibilistic base and $\pi_{\Sigma}$ the possibility distribution induced from $\Sigma$. Let $\langle\alpha, w\rangle$ be any new information, then for any possible world $W$ :

$$
\pi_{\Sigma}\left(\left.W\right|_{r}\langle\alpha, w\rangle\right)=\pi_{\Sigma_{1}}(W)
$$

where $\Sigma_{1}=\Sigma \circledast_{r}\langle\alpha, w\rangle$.

As a direct consequence of the above equivalence result and Theorem 4.2 reinforcement possibilistic base revision also shares most of the nice properties stated in Section 3.2

\section{Related Work and Conclusion}

We have presented a computational model of so-called reinforcement belief revision. This operator has been shown semantically equivalent to an OCF-based model of reinforcement revision. This implies that reinforcement base revision satisfies all desirable rationality postulates but violates those that are too radical. Moreover, we have formally analyzed the time and space complexity of the computational model of reinforcement revision. In this final section, we will give a detailed comparison between our proposal and existing revision operators.

\subsection{Syntax Irrelevant Operators}

In the literature, there are revision operators which do not exploit any explicit extralogical preference information. From a representational point of view, this seems to be an advantage; but these revision operators are also criticized as inflexible [Nebel, 1998], because they allow little control over which sentences are discarded and which are retained. In the following, we present one well-known syntax irrelevant revision operator proposed by [Dalal, 1988]. We will argue that Dalal's operator, while sacrificing flexibility, does not have the benefit of lower time and/or space consumption. As shown in |Eiter and Gottlob, 1992, Cadoli et al., 1995|, the same holds for most other syntax irrelevant operators.

Dalal's operator is based on a notion of distance (between possible worlds): When a belief base $B$ is revised by $\alpha$, the revised belief base should be determined by the models of $\alpha$ that are "closest" to those of $B$ [Dalal, 1988]. 
Formally, the distance between two possible worlds $W_{1}, W_{2}$, denoted by $\|\Delta\|\left(W_{1}, W_{2}\right)$, is the cardinality of their symmetric difference:

$$
\|\Delta\|\left(W_{1}, W_{2}\right)=\left\|\left(W_{1} \backslash W_{2}\right) \cup\left(W_{2} \backslash W_{1}\right)\right\|
$$

The distance between a possible world $W$ and a (consistent) belief base $B$, denoted by $\|\Delta\|^{\min }(B, W)$, is the minimal distance between $W$ and models of $B$ :

$$
\|\Delta\|^{\min }(B, W)=\min \left(\left\{\|\Delta\|\left(W_{1}, W\right) \mid W_{1} \in \operatorname{Mods}(B)\right\}\right)
$$

Given a belief base $B$, we can induce a faithful ranking $\preceq_{C n(B)}$ on $C n(B)$ by stipulating:

$$
W_{1} \preceq_{B} W_{2} \text { iff }\|\Delta\|^{\min }\left(B, W_{1}\right) \leq\|\Delta\|^{\min }\left(B, W_{2}\right)
$$

Dalal's operator is then defined as follows:

$$
C n\left(B *_{d} \alpha\right) \stackrel{\text { def }}{=} \begin{cases}\operatorname{Th}\left(\min \left(\operatorname{Mods}(\alpha), \preceq_{B}\right)\right) & \text { if } B \text { is consistent } \\ \operatorname{Cn}(\{\alpha\}) & \text { otherwise }\end{cases}
$$

It has been shown by [Katsuno and Satoh, 1991] that Dalal's operator satisfies all AGM postulates.

Note that Dalal's operator does not tell us explicitly how to construct a revised belief base. A negative result in Cadoli et al., 1995] says that the size of the revised belief base $B *_{d} \alpha$ can be much larger than that of $B$ and $\alpha$. More precisely, they have shown that if the size of the revised belief base $B *_{d} \alpha$ is polynomially bounded by the size of $B$ and $\alpha$, then $\Sigma_{2}^{p}=\Pi_{2}^{p}=\mathrm{PH}$. As the equality of these classes is highly unlikely, this essentially shows that Dalal's operator causes super-polynomial space explosion. Moreover, as shown in |Eiter and Gottlob, 1992], the problem of CF for Dalal's operator is $\Delta_{2}^{\mathrm{p}}[\mathrm{O}(\log \mathrm{n})]$-complete; however, unlike reinforcement base revision, it remains $\Delta_{2}^{\mathrm{p}}[\mathrm{O}(\log \mathrm{n})]$-complete even for Horn theories.

\subsection{Theory Base Transmutation}

Theory base transmutation is a class of iterated revision operators proposed by |Williams, 1994, among which conditionalization and adjustment are the most prominent ones. Like reinforcement base revision, both conditionalization and adjustment have intuitive OCF-based semantics. We remark that there are two major differences between reinforcement base revision and theory base transmutation: First of all, Postulate (Ind) is violated by both conditionalization and adjustment, and the latter also violates Postulate (DP2); secondly, theory base transmutation allows the input evidence degree to be 0 , and in this case they behave like contraction operators since in theory base transmutation the input evidence degree will be the rank of the input sentence. To facilitate the comparison between reinforcement revision and theory base transmutation, we assume in the following that the input evidence degree is always greater than 0 .

\subsubsection{Conditionalization}

OCF conditionalization was originally introduced in |Spohn, 1988|. It can be viewed as a qualitative version of Jeffrey's Rule of probabilistic conditioning [Goldszmidt, 1992]:

$$
\left(k_{\alpha . m}^{c, *}\right)(W) \stackrel{\text { def }}{=} \begin{cases}k(W)-k(\neg \alpha) & \text { if } W \models \alpha \\ k(W)-k(\alpha)+m & \text { otherwise }\end{cases}
$$




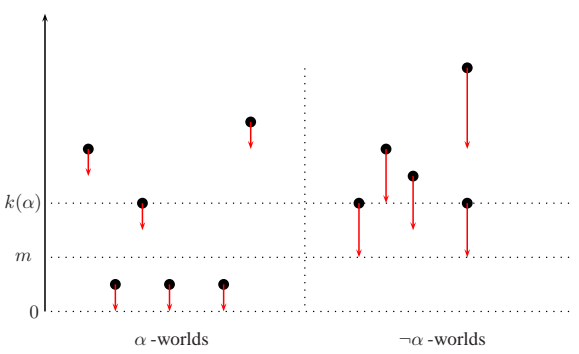

(a) $k(\alpha)>m$

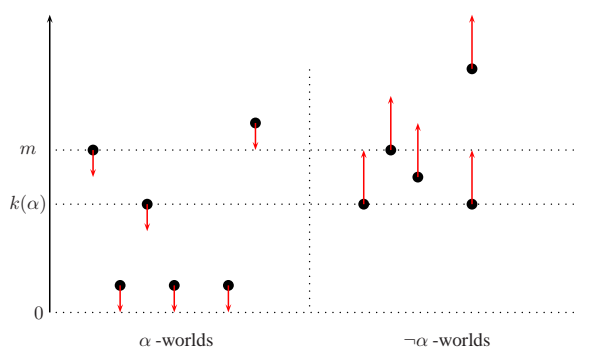

(b) $k(\alpha) \leq m$

Figure 6: OCF conditionalization

It is easy to see that OCF conditionalization closely resembles reinforcement OCF revision. In particular, they coincide if $k(\alpha)=0$. Moreover, the following result shows that OCF conditionalization can in fact be decomposed into a sequence of OCF reinforcement revision steps.

Theorem 5.1. Let $k$ be an $O C F$ and $\langle\alpha, m\rangle$ some new information, then for any possible world $W$ :

$$
k_{\alpha, m}^{c, *}(W)= \begin{cases}k_{\alpha, m}^{r, m-k(\alpha)}(W) & \text { if } k(\alpha)<m \\ \left(\left(k_{\neg \alpha, m^{\prime}}^{r, *}\right)_{\alpha, m}^{r, *}\right)(W) & \text { otherwise }\end{cases}
$$

where $m^{\prime} \in \mathbb{N}^{+}$is an arbitrary positive integer.

The above result shows that OCF reinforcement revision behaves much more subtly than conditionalization.

The intuition behind OCF conditionalization is depicted in Figure 6 where we distinguish the two cases $k(\alpha)>m$ and $k(\alpha) \leq m$. OCF conditionalization changes the ranks of $\alpha$-worlds in exactly the same way as reinforcement OCF revision does. Depending on whether $k(\alpha)>m$ or $k(a) \leq m$, all $\neg \alpha$-worlds are uniformly moved downwards or upwards, respectively, so that the most plausible $\neg \alpha$-worlds end up having the rank $m$, which is necessary to obtain $k_{\alpha, m}^{c, *}(\alpha)=m$. It is easy to prove that OCF conditionalization satisfies (DP1) and (DP2) but violates (Ind) in case $k(\alpha)>m$.

Conditionalization itself is a revision operator on EE-bases defined as follows |Williams, 1992, Benferhat et al., 2002|:

$\langle B, f\rangle *_{c}\langle\alpha, m\rangle \stackrel{\text { def }}{=}\left\{\begin{array}{ll}\langle B, f\rangle *_{r}\langle\alpha, m\rangle & \text { if } B \nvdash \alpha \\ & \left.\cup\left\{\beta, f(\beta)-\operatorname{Rank}_{\Xi}(\alpha)+m\right\rangle \mid f(\beta)>\operatorname{Rank}_{\Xi}(\alpha)\right\} \\ \cup & \{\langle\alpha, m\rangle\}\end{array}\right\}$ otherwise

Note that conditionalization coincides with reinforcement base revision in case $B \nvdash \alpha$. It is not difficult to see that $\Xi *_{r} \alpha \equiv \Xi *_{c} \alpha$ for any EE base $\Xi$ and new information $\langle\alpha, m\rangle$. Therefore, the complexity of conditionalization is same as that of reinforcement base revision.

Theorem 5.2. For conditionalization, the problem of $C F$ is $\Delta_{2}^{\mathrm{p}}[\mathrm{O}(\log \mathrm{n})]$-complete. 


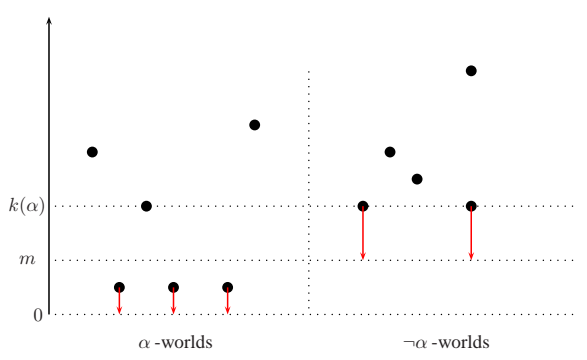

(a) $k(\alpha)>m$

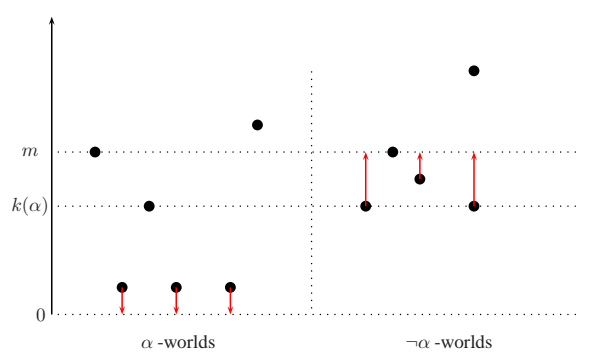

(b) $k(\alpha) \leq m$

Figure 7: OCF adjustment

However, since conditionalization violates Postulates (Ind), it is easy to find counterexamples. Recall, for instance, Example 1 Suppose that now $\Xi=$ $\{\langle r, 6\rangle,\langle s, 5\rangle,\langle r \vee s, 7\rangle\}$ and we first learn that $X$ is smart with evidence degree 1. This yields $\Xi_{1}=\Xi *_{c}\langle s, 1\rangle=\{\langle r \vee s, 3\rangle,\langle r \vee \neg s, 6\rangle,\langle s, 1\rangle\}$. Next we learn that $X$ is not rich with evidence degree 2 , and obtain $\Xi_{2}=\Xi_{1} *_{c}\langle\neg r, 2\rangle=$ $\{\langle r \vee \neg s, 3\rangle,\langle\neg r, 2\rangle,\langle s \vee \neg r, 3\rangle\}$. Hence, contrary to the intuition, we now believe that $X$ is not smart, since $\operatorname{Rank}_{\Xi_{2}}(\neg s)=2$.

\subsubsection{Adjustment}

OCF adjustment is an operator based on an absolute measure of minimal change [Williams, 1994]:

$$
\left(k_{\alpha . m}^{j, *}\right)(W) \stackrel{\text { def }}{=} \begin{cases}\left(k_{\alpha}^{-}\right)_{\alpha, m}^{\times}(W) & \text { if } m<k(\alpha) \\ k_{\alpha, m}^{\times}(W) & \text { otherwise }\end{cases}
$$

where

$$
\begin{gathered}
\left(k_{\alpha}^{-}\right)(W)= \begin{cases}0 & \text { if } W \models \neg \alpha \text { and } K(W)=k(\alpha) \\
k(W) & \text { otherwise }\end{cases} \\
\left(k_{\alpha, m}^{\times}\right)(W)= \begin{cases}0 & \text { if } W \models \alpha \text { and } K(W)=k(\neg \alpha) \\
i & \text { else if } W \models \neg \alpha \text { and } K(W)<i \\
k(W) & \text { else }\end{cases}
\end{gathered}
$$

Although the definition seems quite complicated, the graphical representation in Figure 7 provides a nice intuition of OCF adjustment. Unlike OCF conditionalization and reinforcement OCF revision, OCF adjustment only changes the ranks of the most plausible $\alpha$-worlds and $\neg \alpha$-worlds. Moreover, when $k(a) \leq m$, the relative plausibility ordering of $\neg \alpha$-worlds is not always preserved (cf. Figure 7 b)). It is easy to verify that OCF adjustment satisfies only Postulate (DP1), but violates (DP2) and (Ind).

Adjustment is an EE-base revision operator defined as follows |Williams, 1992 
Benferhat et al., 2002:

$$
\langle B, f\rangle *_{j}\langle\alpha, m\rangle=\left\{\begin{array}{l}
\left\{\langle\beta, f(\beta)\rangle \mid f(\beta)>\operatorname{Rank}_{\Xi}(\neg \alpha)\right\} \\
\cup\left\{\langle\alpha \vee \beta, f(\beta)\rangle \mid m<f(\beta) \leq \operatorname{Rank}_{\Xi}(\neg \alpha)\right\} \\
\cup\{\langle\alpha, m\rangle\} \\
\left\{\langle\beta, f(\beta)\rangle \mid f(\beta)>\operatorname{Rank}_{\Xi}(\alpha)\right\} \\
\cup\left\{\langle\neg \alpha \vee \beta, f(\beta)\rangle \mid f(\beta) \leq \operatorname{Rank}_{\Xi}(\alpha)\right\} \\
\cup\{\langle\alpha, m\rangle\}
\end{array}\right\} \text { if } B \nvdash \alpha
$$

Just like conditionalization, adjustment has the same complexity as reinforcement base revision:

Theorem 5.3. For adjustment, the problem of $C F$ is $\Delta_{2}^{\mathrm{p}}[\mathrm{O}(\log \mathrm{n})]$-complete.

Similar to conditionalization, it is easy to find examples in which adjustment leads to counter-intuitive conclusions. Consider, for instance, Example 1 again. Suppose that $\Xi=\left(\emptyset *_{r}\langle r, 1\rangle\right) *_{r}\langle s, 1\rangle=\{\langle r, 1\rangle,\langle s, 1\rangle,\langle r \vee s, 2\rangle\}$ and we first learn that $X$ is not smart with evidence degree 2 , followed by learning the opposite with the same evidence degree. With adjustment we obtain the revised EE-bases $\Xi_{1}=\Xi *_{j}\langle\neg s, 2\rangle=$ $\{\langle r \vee s, 2\rangle,\langle\neg s, 2\rangle\}$ and $\Xi_{2}=\Xi_{1} *_{j}\langle s, 2\rangle=\{\langle s, 2\rangle\}$. Hence, contrary to the intuition, we do not believe that $X$ is rich anymore.

\section{Acknowledgments}

The authors are grateful to the anonymous reviewers of an earlier version of this paper for helpful comments and suggestions. 


\section{A Proofs}

Proposition 3.2. Suppose that $\Xi=\langle B, f\rangle$ is an EE base and $k_{\Xi}$ the induced OCF as defined by (12), then for any sentence $\beta$ :

$$
\operatorname{Rank}_{\Xi}(\beta)=k_{\Xi}(\beta)
$$

Proof. Assume $\vdash \beta$. Then $\operatorname{Rank}_{\Xi}(\beta)=k_{\Xi}(\beta)=\infty$.

Assume $\nvdash \beta$ and $\operatorname{Rank}_{\Xi}(\beta)=i$. It follows from (4) that $\Xi^{i+1} \nvdash \beta$ and $\Xi^{i} \vdash \beta$. Let $W$ be a possible world such that $W \models \Xi^{i+1} \cup\{\neg \beta\}$. From $\Xi^{i} \vdash \beta$, it follows that $W \forall \forall \Xi^{i}$. Therefore, there must be a sentence $\left.\beta_{i} \in \Xi\right|_{i}$ such that $W \not \forall \beta_{i}$. It follows from (12) that $k_{\Xi}(W)=i$. Let $W_{1}$ be any possible world such that $k_{\Xi}\left(W_{1}\right)<k_{\Xi}(W)$. It follows from (12) that $W_{1} \models \Xi^{i}$; hence, $W_{1} \models \beta$. According to 9, we have $k_{\Xi}(\beta)=i$.

Theorem 3.3. Suppose that $\Xi$ is an EE base and $k_{\Xi}$ the OCF induced from $\Xi$. Let $\langle\alpha, m\rangle$ be any new information, then for all possible worlds $W$ :

$$
k_{\Xi_{1}}(W)=k_{\Xi_{\alpha, m}}^{r, *}(W)
$$

where $\Xi_{1}=\Xi *_{r}\langle\alpha, m\rangle$.

Proof. Let $k_{\Xi}(W)=i$. By 12, we have $W \models \Xi^{i+1}$ and there exists a sentence $\left.\beta_{i} \in \Xi\right|_{i}$ such that $W \models \neg \beta_{i}$.

If $W \models \neg \alpha$, then (11) implies that $k_{\Xi_{\alpha, m}^{r, *}}^{r, W)}(W+m$. It follows from (7) that $\Xi_{1}^{i+m+1}=\Xi^{i+m+1} \cup\left\{\beta \vee \alpha \mid \beta \in \Xi^{i+1}\right\}$ and $\left.\alpha \vee \beta_{i} \in \Xi_{1}\right|_{i+m}$. Obviously, we have $W \models \Xi_{1}^{i+m+1}$ and $W \forall \alpha \vee \beta_{i}$. Hence, according to 12], $k_{\Xi_{1}}(W)=i+m$.

If $W \models \alpha$, then (11) implies that $k_{\Xi_{\alpha, m}}^{r, *}(W)=i-k_{\Xi}(\neg \alpha)$. Let $\operatorname{Rank}_{\Xi}(\neg \alpha)=$ $r$. It follows from (7) that $\Xi_{1}^{i-r+1} \subseteq \Xi^{i+1} \cup\{\beta \vee \alpha \mid \beta \in B\} \cup\{\alpha\}$ and $\left.\beta_{i} \in \Xi_{1}\right|_{i-r}$. It is obvious that $W \models \Xi_{1}^{i-r+1}$. It follows from (12) that $k_{\Xi_{1}}(W)=i-r=$ $i-\operatorname{Rank}_{\Xi}(\neg \alpha)$. According to Proposition 3.2 we obtain that $k_{\Xi_{1}}(W)=i-k_{\Xi}(\neg \alpha)$.

Some of the proofs below rely on the following lemma.

Lemma A.1. Suppose that $\Xi$ is an EE base and $\langle\alpha, m\rangle$ is any new information. Let $\Xi_{1}=\Xi *_{r}\langle\alpha, m\rangle$, then for any non-tautologous sentence $\beta$ :

$$
\operatorname{Rank}_{\Xi_{1}}(\beta)= \begin{cases}t+m & \text { if } \vdash \alpha \rightarrow \beta \\ t^{\prime}-\bar{r} & \text { else if } t^{\prime}=t \\ \min \left(t^{\prime}-\bar{r}, t+m\right) & \text { otherwise }\end{cases}
$$

where $\bar{r}=\operatorname{Rank}_{\Xi}(\neg \alpha), t=\operatorname{Rank}_{\Xi}(\beta)$, and $t^{\prime}=\operatorname{Rank}_{\Xi}(\alpha \rightarrow \beta)$. 
Proof. This is a direct consequence of the following property, whose proof can be found in [Jin and Thielscher, 2007], and Theorem 3.3. Let $k$ be an OCF and $\langle\alpha, m\rangle$ any new information, then for any non-tautological sentence $\beta$,

$$
k_{\alpha, m}^{r, *}(\beta)= \begin{cases}k(\beta)+m & \text { if } \vdash \alpha \rightarrow \beta \\ k(\alpha \rightarrow \beta)-k(\neg \alpha) & \text { else if } k(\alpha \rightarrow \beta)=k(\beta) \\ \min (k(\alpha \rightarrow \beta)-k(\neg \alpha), k(\beta)+m) & \text { otherwise }\end{cases}
$$

Proposition 3.1. Suppose that $\Xi_{1}$ is the result of revising an EE base $\Xi$ by $\langle\neg \alpha, m\rangle$, where $\alpha$ is an arbitrary sentence and $m$ an arbitrary natural number. Then for any sentence $\beta$ :

$$
\operatorname{Rank}_{\Xi_{1}}(\beta) \neq \operatorname{Rank}_{\Xi}(\beta) \text { iff } \operatorname{Rank}_{\Xi_{2}}(\alpha) \neq \operatorname{Rank}_{\Xi}(\alpha)
$$

where $\Xi_{2}=\Xi *_{r}\left\langle\neg \beta, m^{\prime}\right\rangle$ for an arbitrary natural number $m^{\prime}$.

Proof. Assume, without loss of generality, that $\operatorname{Rank}_{\Xi_{1}}(\beta)=\operatorname{Rank}_{\Xi}(\beta)$ and $\operatorname{Rank}_{\Xi_{2}}(\alpha) \neq \operatorname{Rank}_{\Xi}(\alpha)$. From Lemma A.1 it follows that $\nvdash \neg \alpha \rightarrow \beta$ and $\operatorname{Rank}_{\Xi}(\beta)=\operatorname{Rank}_{\Xi_{1}}(\beta)=\operatorname{Rank}_{\Xi}(\neg \alpha \rightarrow \beta)-\operatorname{Rank}_{\Xi}(\alpha)$. Therefore, $\operatorname{Rank}_{\Xi}(\alpha)=$ $\operatorname{Rank}_{\Xi}(\neg \alpha \rightarrow \beta)-\operatorname{Rank}_{\Xi}(\beta)$. Note that $\operatorname{Rank}_{\Xi}(\neg \alpha \rightarrow \beta)=\operatorname{Rank}_{\Xi}(\neg \beta \rightarrow \alpha)$. Therefore, Lemma A.1 implies that $\operatorname{Rank}_{\Xi_{2}}(\alpha)=\operatorname{Rank}_{\Xi}(\neg \alpha \rightarrow \beta)-\operatorname{Rank}_{\Xi}(\beta)$, which contradicts $\operatorname{Rank}_{\Xi_{2}}(\alpha) \neq \operatorname{Rank}_{\Xi}(\alpha)$.

Proposition 3.7 Let $\Xi_{1}, \Xi_{2}$ be two epistemically equivalent EE bases, then for any sentence $\alpha$ and evidence degrees $m_{1}, m_{2} \in \mathbb{N}^{+}$:

$$
\operatorname{Bel}\left(\Xi_{1}^{\prime}\right)=\operatorname{Bel}\left(\Xi_{2}^{\prime}\right)
$$

where $\Xi_{1}^{\prime}=\Xi_{1} *_{r}\left\langle\alpha, m_{1}\right\rangle$ and $\Xi_{2}^{\prime}=\Xi_{2} *_{r}\left\langle\alpha, m_{2}\right\rangle$.

Proof. Let $\beta \in \operatorname{Bel}\left(\Xi_{1}^{\prime}\right)$. It follows that $\operatorname{Rank}_{\Xi_{1}^{\prime}}(\beta)>0$. According to Lemma A.1 it must then be the case that either $\vdash \alpha \rightarrow \beta$ or $\operatorname{Rank}_{\Xi_{1}}(\alpha \rightarrow \beta)>\operatorname{Rank}_{\Xi_{1}}(\neg \alpha)$.

If $\vdash \alpha \rightarrow \beta$, then according to Lemma A.1 we have that $\operatorname{Rank}_{\Xi_{2}^{\prime}}(\beta)=$ $\operatorname{Rank}_{\Xi_{2}}(\beta)+m_{2}>0$.

If $\operatorname{Rank}_{\Xi_{1}}(\alpha \rightarrow \beta)>\operatorname{Rank}_{\Xi_{1}}(\neg \alpha)$, then also $\operatorname{Rank}_{\Xi_{2}}(\alpha \rightarrow \beta)>\operatorname{Rank}_{\Xi_{2}}(\neg \alpha)$ since $\Xi_{2}$ is epistemically equivalent to $\Xi_{1}$. Again, it follows from Lemma A.1 that $\operatorname{Rank}_{\Xi_{2}^{\prime}}(\beta)>\operatorname{Rank}_{\Xi_{1}}(\alpha \rightarrow \beta)-\operatorname{Rank}_{\Xi_{1}}(\neg \alpha)>0$.

The above discussion shows that $\operatorname{Bel}\left(\Xi_{1}^{\prime}\right) \subseteq \operatorname{Bel}\left(\Xi_{2}^{\prime}\right)$. The converse, $\operatorname{Bel}\left(\Xi_{2}^{\prime}\right) \subseteq$ $\operatorname{Bel}\left(\Xi_{1}^{\prime}\right)$, can be shown symmetrically.

Proposition 3.8 Let $\Xi_{1}, \Xi_{2}$ be two equivalent EE bases and $\langle\alpha, m\rangle$ some new information, then

$$
\Xi_{1} *_{r}\langle\alpha, m\rangle \cong \Xi_{2} *_{r}\langle\alpha, m\rangle
$$

Proof. A direct consequence of Lemma A.1 
Theorem 3.9. For reinforcement base revision, the problem of $C F$ is $\Delta_{2}^{\mathrm{p}}[\mathrm{O}(\log \mathrm{n})]$ complete.

Proof. We first show that the problem is in $\Delta_{2}^{\mathrm{p}}[\mathrm{O}(\log \mathrm{n})]$. It is easy to see that in order to compute a revised EE base $\Xi_{1}=\Xi *_{r}\langle\alpha, m\rangle$, we mainly need to calculate $\operatorname{Rank}_{\Xi}(\neg \alpha)$ (cf. Algorithm 2 in Appendix B. Algorithm 1 in Appendix B shows that $\operatorname{Rank}_{\Xi}(\neg \alpha)$ can be computed with at most logarithmic many NP-oracle calls. Once the revised EE base $\Xi_{1}$ has been computed, we just need one additional NP-oracle to decide whether $\Xi_{1}$ entails $\beta$. Therefore, the problem is in $\Delta_{2}^{\mathrm{p}}[\mathrm{O}(\log \mathrm{n})]$.

To show that the problem is $\Delta_{2}^{\mathrm{p}}[\mathrm{O}(\log \mathrm{n})]$-hard, we give a polynomial (many-toone) reduction from the problem of $\mathrm{CF}$ for cut base revision (which is $\Delta_{2}^{\mathrm{p}}[\mathrm{O}(\log \mathrm{n})]$ hard [Nebel, 1994|). Given any prioritized base $\left\langle B, \leq_{B}\right\rangle$, we can construct a EE base $\Xi=\langle B, f\rangle$ by assigning evidence degree 1 to all sentences in the lowest class and evidence degree 2 to all sentences in the next higher class, and so on. It is easy to see that for any sentences $\alpha$ and $\beta:\left\langle B, \leq_{B}\right\rangle *_{c u t} \alpha \vdash \beta$ iff $\Xi_{1}$ entails $\beta$ where $\Xi_{1}=\Xi *_{r}\langle\alpha, 1\rangle$. Thus, it follows that the problem of CF for reinforcement base revision is $\Delta_{2}^{\mathrm{p}}[\mathrm{O}(\log \mathrm{n})]$-hard.

Theorem 3.10. For reinforcement base revision, the problem of computing a revised belief state is NP-equivalent.

Proof. The proof of Theorem 3.9 shows that the problem is NP-easy. To show that it is also NP-hard, it suffices to observe that the problem of satisfiability of an arbitrary sentence $\beta$ can be reduced to the problem of computing the revision $\{\langle\beta, 1\rangle\} *_{r}\langle T, 1\rangle$ : According to (7), the revised belief base includes $\langle\beta, 1\rangle$ iff $\operatorname{cut}_{\{\langle\beta, 1\rangle\}}(\perp)=\{\beta\}$ and $\operatorname{Rank}_{\{\langle\beta, 1\rangle\}}(\perp)=0$. The latter is equivalent to $\beta$ being satisfiable according to (5) and (4).

Proposition 4.1 Consider a possibility distribution $\pi$ revised by a new nontautological evidence $\alpha$ with possibility degree $w$. Let $\pi_{1}=\pi_{\langle\alpha, w\rangle}^{*}$, then

$$
N_{\pi_{1}}(\alpha)=N_{\pi}(\alpha)+w-w \times N_{\pi}(\alpha)
$$

Proof. Assume $N_{\pi}(\alpha)=1-\Pi_{\pi}(\neg \alpha)=w^{\prime}$. According to (14, we have $\max (\{\pi(W) \mid W \models \neg \alpha\})=1-w^{\prime}$. From 17, it follows that $\max \left(\left\{\pi_{1}(W) \mid W \models\right.\right.$ $\neg \alpha\})=\left(1-w^{\prime}\right) \times(1-w)$. Then (14) implies $\Pi_{\pi_{1}}(\neg \alpha)=\left(1-w^{\prime}\right) \times(1-w)$. Thus, according to [15, we have $N_{\pi_{1}}(\alpha)=1-\left(1-w^{\prime}\right) \times(1-w)=w+w^{\prime}-w \times w^{\prime}$.

The following observation is needed for the proof of Theorem 4.2

Lemma A.2. Let $k$ be an $O C F$, and $\mathrm{P}(k)$ the possibility distribution as defined by 116, then for any sentence $\alpha$ :

$$
\Pi_{\pi_{k}}(\alpha)=e^{-k(\neg \alpha)}
$$

Proof. Recall that $\Pi_{\pi}(\alpha)$ is defined as $\max (\{\pi(W) \mid W \models \alpha\})$. Let $W_{1}$ be a possible world such that $\pi_{k}\left(W_{1}\right)=\Pi_{\pi_{k}}(\alpha)=\max \left(\left\{\pi_{k}(W) \mid W \models \alpha\right\}\right)$. Since $f(x)=e^{-x}$ is monotonically decreasing, we have $k\left(W_{1}\right)=\min (\{k(W) \mid W \models$ $\alpha\})$. It follows from (9) that $k\left(W_{1}\right)=k(\neg \alpha)$. Thus $\Pi_{\pi_{k}}(\alpha)=e^{-k(\neg \alpha)}$. 
Theorem 4.2 Suppose that $k$ is an OCF and $\langle\alpha, m\rangle$ (with $m \in \mathbb{N}^{+}$) some input information. Let $k_{\langle\alpha, m\rangle}^{*}$ be the revised OCF using reinforcement OCF revision, then for any possible world $W$ :

$$
\pi_{k_{\langle\alpha, m\rangle}^{*}}(W)=\pi_{k}\left(\left.W\right|_{r}\left\langle\alpha, 1-e^{-m}\right\rangle\right)
$$

Proof. Assume $W \models \alpha$. According to $16, \pi_{k_{\langle\alpha, m\rangle}^{*}}(W)=e^{-k_{\alpha, m}^{*}(W)}$. It follows from 111 that $e^{-k_{\alpha, m}^{*}(W)}=e^{-(k(w)-k(\neg \alpha))}$. On the other hand, 17 implies $\pi_{k}\left(W \mid{ }_{r}\left\langle\alpha, 1-e^{-m}\right\rangle\right)=\frac{\pi_{k}(W)}{\Pi_{\pi_{k}}(\alpha)}$. It follows from 16 and Lemma A.2 that $\pi_{k}\left(\left.W\right|_{r}\left\langle\alpha, 1-e^{-m}\right\rangle\right)=\frac{e^{-k(w)}}{e^{-k(\neg \alpha)}}$. Hence $\pi_{k_{\langle\alpha, m\rangle}^{*}}(W)=\pi_{k}\left(\left.W\right|_{r}\left\langle\alpha, 1-e^{-m}\right\rangle\right)$.

Assume $W \forall \alpha$. Similar to the above, according to (16) and 11], $\pi_{k_{\langle\alpha, m\rangle}^{*}}(W)=$ $e^{-(k(W)+m)}$. It follows from (17) that $\pi_{k}\left(\left.W\right|_{r}\left\langle\alpha, 1-e^{-m}\right\rangle\right)=\pi_{k}(W) \times e^{-m}$. (16) implies $\pi_{k}\left(\left.W\right|_{r}\left\langle\alpha, 1-e^{-m}\right\rangle\right)=e^{-k(W)} \times e^{-m}$. Hence $\pi_{k_{\langle\alpha, m\rangle}^{*}}(W)=$ $\pi_{k}\left(\left.W\right|_{r}\left\langle\alpha, 1-e^{-m}\right\rangle\right)$.

Theorem 4.4 Suppose that $\Sigma$ is a possibilistic base and $\pi_{\Sigma}$ the possibility distribution induced from $\Sigma$. Let $\langle\alpha, w\rangle$ be any new information, then for any possible world $W$ :

$$
\pi_{\Sigma}\left(\left.W\right|_{r}\langle\alpha, w\rangle\right)=\pi_{\Sigma_{1}}(W)
$$

where $\Sigma_{1}=\Sigma \Theta_{r}\langle\alpha, w\rangle$.

Proof. Analogous to the proof of Theorem 3.3

Theorem 5.1 Let $k$ be an OCF and $\langle\alpha, m\rangle$ some new information, then for any possible world $W$ :

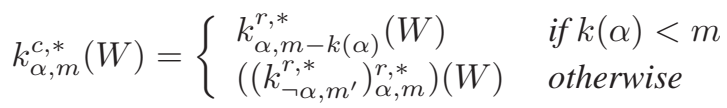

where $m^{\prime} \in \mathbb{N}^{+}$is an arbitrary positive integer.

Proof.

Assume $k(\alpha)<m$, then it follows directly from 21 and 11 that $k_{\alpha, m}^{c, *}(W)=$ $k_{\alpha, m-k(\alpha)}^{r, *}(W)$.

Assume $k(\alpha) \geq m$. Suppose $W \models \alpha$. According to (21), $k_{\alpha, m}^{c, *}(W)=k(W)-$ $k(\neg \alpha)$, whereas 111 implies that $\left(\left(k_{\neg \alpha, m^{\prime}}^{r, *}\right)_{\alpha, m}^{r, *}\right)(W)=k_{\neg \alpha, m^{\prime}}^{r, *}(W)-k_{\neg \alpha, m^{\prime}}^{r, *}(\neg \alpha)$. Again, 111 implies that $k_{\left\langle\neg \alpha, m^{\prime}\right\rangle}^{r, *}(W)=k(W)+m^{\prime}$. From item 3 of Theorem 3.5] it follows that $k_{\neg \alpha, m^{\prime}}^{r, *}(\neg \alpha)=k(\neg \alpha)+m^{\prime}$. Therefore, $\left(\left(k_{\neg \alpha, m^{\prime}}^{r, *}\right)_{\alpha, m}^{r, *}\right)(W)=k(W)-$ $k(\neg \alpha)$. Thus, $\left(\left(k_{\neg \alpha, m^{\prime}}^{r, *}\right)_{\alpha, m}^{r, *}\right)(W)=k_{\langle\alpha, m\rangle}^{c, *}(W)$.

Suppose $W \forall \forall \alpha$. It follows from (21) that $k_{\alpha, m}^{c, *}(W)=k(W)-k(\alpha)+m$, whereas (11) implies that $\left(\left(k_{\neg \alpha, m^{\prime}}^{r, *}\right)_{\alpha, m}^{r, *}\right)(W)=k_{\neg \alpha, m^{\prime}}^{r, *}(W)+m$. According to 11, $k_{\neg \alpha, m^{\prime}}^{r, *}(W)=k(W)-k(\alpha)$. Therefore, $\left(\left(k_{\neg \alpha, m^{\prime}}^{r, *}\right)_{\alpha, m}^{r, *}\right)(W)=k(W)-k(\alpha)+m$. Thus, $\left(\left(k_{\neg \alpha, m^{\prime}}^{r, *}\right)_{\alpha, m}^{r, *}\right)(W)=k_{\langle\alpha, m\rangle}^{c, *}(W)$. 
Theorem 5.2 For conditionalization, the problem of $C F$ is $\Delta_{2}^{\mathrm{p}}[\mathrm{O}(\log \mathrm{n})]$-complete.

Proof. It suffices to show that $\Xi *_{r} \alpha \equiv \Xi *_{c} \alpha$ for any EE base $\Xi$ and new information $\langle\alpha, m\rangle$. If $B \nvdash \alpha$, this holds trivially. Assume $B \vdash \alpha$. Then $\Xi *_{r} \alpha \equiv \Xi \equiv \Xi *_{c} \alpha$, since both conditionalization and reinforcement base revision satisfy the AGM postulates.

Theorem 5.3. For adjustment, the problem of CF is $\Delta_{2}^{\mathrm{p}}[\mathrm{O}(\log \mathrm{n})]$-complete.

Proof. It suffices to show that $\Xi *_{r} \alpha \equiv \Xi *_{j} \alpha$ for any EE base $\Xi$ and new information $\langle\alpha, m\rangle$. Assume $B \vdash \alpha$. Then $\Xi *_{r} \alpha \equiv \Xi \equiv \Xi *_{j} \alpha$, since both adjustment and reinforcement base revision satisfy the AGM postulates. Assume $B \nvdash \alpha$. The only difference between $\Xi *_{r} \alpha$ and $\Xi *_{j} \alpha$ is that the former contains additional sentences of the form $\alpha \vee \beta_{i}$. Since both revised bases contain $\alpha$, it follows that $\Xi *_{r} \alpha \equiv \Xi \equiv \Xi *_{j} \alpha$. 


\section{B Algorithms}

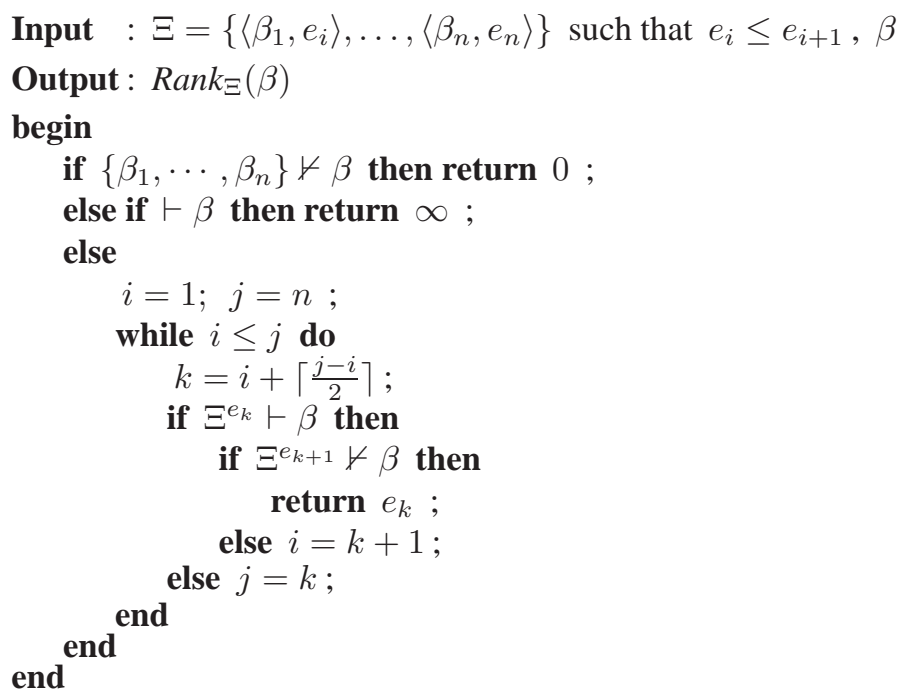

Algorithm 1: Computing $\operatorname{Rank}_{\Xi}(\beta)$

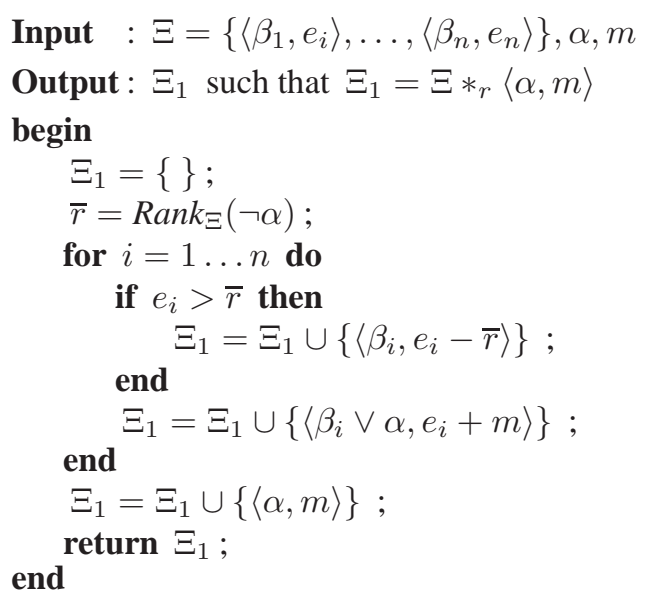

Algorithm 2: Reinforcement base revision 


\section{References}

[Alchourrón and Makinson, 1985] C. E. Alchourrón and D. Makinson. On the logic of theory change: Safe contraction. Studia Logica, 44, pages 405-422, 1985.

[Alchourrón et al., 1985] C. E. Alchourrón, P. Gärdenfors, and D. Makinson. On the logic of theory change: Partial meet contraction and revision functions. The Journal of Symbolic Logic 50(2), pages 510-530, 1985.

[Benferhat et al., 2002] S. Benferhat, D. Dubois, H. Prade, and M.-A. Williams. A practical approach to revising prioritized knowledge bases. Studia Logica, 70(1):105-130, 2002.

[Boutilier, 1993] C. Boutilier. Revision sequences and nested conditionals. In Proceedings of the International Joint Conference on Artificial Intelligence (IJCAI), pages 519-525, 1993.

[Cadoli et al., 1995] M. Cadoli, F. M. Donini, P. Liberatore, and M. Schaerf. The size of a revised knowledge base. In PODS '95: Proceedings of the fourteenth ACM SIGACT-SIGMOD-SIGART symposium on Principles of database systems, pages 151-162, New York, NY, USA, 1995. ACM Press.

[Dalal, 1988] M. Dalal. Updates in propositional databases. Technical Report DCSTR 222, Rutgers University, Dept. of Computer Science, Feb 1988.

[Darwiche and Pearl, 1997] A. Darwiche and J. Pearl. On the logic of iterated belief revision. Artificial Intelligence 89, 1-29, 1997.

[Dixon, 1994] S. E. Dixon. Belief revision: A computational approach. PhD thesis, Basser Department of Computer Science, University of Sydney, Australia, 1994.

[Dubois and Prade, 1991] D. Dubois and H. Prade. Epistemic entrenchment and possibilistic logic. Artificial Intelligence, 50(2):223-239, 1991.

[Dubois and Prade, 1992] D. Dubois and H. Prade. Belief change and possibility theory. In P. Gärdenfors, editor, Belief Revision, pages 142-182. Cambridge University Press, 1992.

[Dubois et al., 1994] D. Dubois, J. Lang, and H. Prade. Possibilistic logic. In Handbook of logic in artificial intelligence and logic programming (vol. 3): nonmonotonic reasoning and uncertain reasoning, pages 439-513. Oxford University Press, Inc., New York, NY, USA, 1994.

[Dubois et al., 1998] D. Dubois, S. Moral, and H. Prade. Belief change rules in ordinal and numerical uncertainty theories. In D. M. Gabbay and P. Smets, editors, Handbook of Defeasible Reasoning and Uncertainty Management Systems, volume 3, pages 311-392. Kluwer Academic Publishers, Dordrecht, 1998.

[Eiter and Gottlob, 1992] T. Eiter and G. Gottlob. On the complexity of propositional knowledge base revision, updates, and counterfactuals. Artificial Intelligence, 57:227-270, 1992.

[Gärdenfors and Makinson, 1988] P. Gärdenfors and D. Makinson. Revisions of knowledge systems using epistemic entrenchment. In Proceedings of the Second Conference on Theoretical Aspects of Reasoning about Knowledge (TARK), pages 83-95, Asilomar, CA, 1988. 
[Gärdenfors, 1988] P. Gärdenfors. Knowledge in Flux: Modeling the Dynamics of Epistemic States. MIT Press, 1988.

[Goldszmidt, 1992] M. Goldszmidt. Qualitative probabilities: a normative framework for commonsense reasoning. $\mathrm{PhD}$ thesis, University of California at Los Angeles, 1992.

[Hansson, 2003] S. O. Hansson. Ten philosophical problems in belief revision. Journal of Logic Computation, 13(1):37-49, 2003.

[Jeffrey, 1965] R. C. Jeffrey. The logic of decision. New York, 1965.

[Jin and Thielscher, 2007] Y. Jin and M. Thielscher. Iterated belief revision, revised. Artificial Intelligence, 171(1):1-18, 2007.

[Katsuno and Mendelzon, 1991] H. Katsuno and A. O. Mendelzon. Propositional knowledge base revision and minimal change. Artificial Intelligence, 52(3):263294, 1991.

[Katsuno and Satoh, 1991] H. Katsuno and K. Satoh. A unified view of consequence relation, belief revision and conditional logic. In Proceedings of the International Joint Conference on Artificial Intelligence (IJCAI), pages 406-412, 1991.

[Konieczny and Pérez, 2000] S. Konieczny and R. P. Pérez. A framework for iterated revision. Journal of Applied Non-Classical Logics, 10(3-4), 2000.

[Liberatore, 1997a] P. Liberatore. The complexity of belief update. In Proceedings of the Fifteenth International Joint Conference on Artificial Intelligence (IJCAI'97), pages 68-73, 1997.

[Liberatore, 1997b] P. Liberatore. The complexity of iterated belief revision. In F. N. Afrati and P. G. Kolaitis, editors, Proceeding of the 6th International Conference on Database Theory (ICDT), pages 276-290, 1997.

[Nayak et al., 1996] A. Nayak, N. Foo, M. Pagnucco, and A. Sattar. Changing conditional belief unconditionally. In Proceedings of the Sixth Conference on Theoretical Aspects of Rationality and Knowledge (TARK), pages 119-135. Morgan Kaufmann Publishers Inc., San Francisco, CA, USA, 1996.

[Nayak et al., 2003] A. Nayak, M. Pagnucco, and P. Peppas. Dynamic belief revision operators. Artificial Intelligence, 146(2):193-228, 2003.

[Nayak, 1994] A. Nayak. Iterated belief change based on epistemic entrenchment. Erkenntnis, 4:353-390, 1994.

[Nebel, 1991] B. Nebel. Belief revision and default reasoning: Syntax-based approaches. In J. A. Allen, R. Fikes, and E. Sandewall, editors, Principles of Knowledge Representation and Reasoning: Proceedings of the 2nd International Conference, pages 417-428, San Mateo, 1991. Morgan Kaufmann.

[Nebel, 1992] B Nebel. Syntax-based approaches to belief revision. In P. Gärdenfors, editor, Belief Revision, volume 29, pages 52-88. Cambridge University Press, Cambridge, UK, 1992. 
[Nebel, 1994] B. Nebel. Base revision operations and schemes: Semantics, representation and complexity. In Proceedings of the European Conference on Artificial Intelligence (ECAI), pages 341-345, 1994.

[Nebel, 1998] B. Nebel. How hard is it to revise a belief base? In Didier Dubois and Henri Prade, editors, Handbook of Defeasible Reasoning and Uncertainty Management Systems, Volume 3: Belief Change, pages 77-145. Kluwer Academic Publishers, Dordrecht, 1998.

[Newell, 1982] A. Newell. The knowledge level. Artificial Intelligence, 18:87-127, 1982.

[Papadimitriou, 1994] C. Papadimitriou. Computational Complexity. AddisonWesley, 1994.

[Spohn, 1988] W. Spohn. Ordinal conditional functions: A dynamic theory of epistemic state. In W. L. Harper and B. Skyrms, editors, Causation in Decision: Belief, Change and Statistics: Proceedings of the Irvine Conference on Probability and Causation, volume II, pages 105-134, Dordrecht, 1988. Kluwer Academic Publisher.

[Spohn, 1991] W. Spohn. A reason for explanation: Explanations provide stable reasons. In W. Spohn et al., editor, Existence and Explanation, pages 165-196. Kluwer Academic Publisher, 1991.

[Wassermann, 1999] R. Wassermann. Resource-Bounded Belief Revision. PhD thesis, ILLC, University of Amsterdam, 1999.

[Williams, 1992] M. Williams. Two operators for theory base change. In Proceedings of the Fifth Australian Joint Conference on Artificial Intelligence, pages 259-265, 1992.

[Williams, 1994] M.-A. Williams. Transmutations of knowledge systems. In J. Doyle, E. Sandewall, and P. Torasso, editors, Principles of Knowledge Representation and Reasoning (KR), pages 619-629, Bonn, Germany, 1994. Morgan Kaufmann.

[Wobcke, 1992] W. R. Wobcke. On the use of epistemic entrenchment in nonmonotonic reasoning. In Proceedings of the Tenth European Conference on Artificial Intelligence (ECAI), pages 324-328, 1992. 